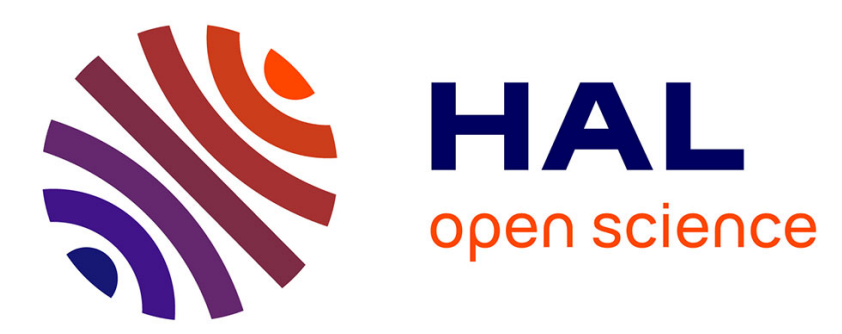

\title{
All-optical signal processing based on self-induced polarization control in optical fibers
}

Massimiliano Guasoni, P.-Y. Bony, Marin Gilles, Antonio Picozzi, Julien Fatome

\section{- To cite this version:}

Massimiliano Guasoni, P.-Y. Bony, Marin Gilles, Antonio Picozzi, Julien Fatome. All-optical signal processing based on self-induced polarization control in optical fibers. Journal of Lightwave Technology, 2015, 34 (2), pp.327-341. 10.1109/JLT.2015.2498206 . hal-01285323

\section{HAL Id: hal-01285323 \\ https://hal.science/hal-01285323}

Submitted on 9 Mar 2016

HAL is a multi-disciplinary open access archive for the deposit and dissemination of scientific research documents, whether they are published or not. The documents may come from teaching and research institutions in France or abroad, or from public or private research centers.
L'archive ouverte pluridisciplinaire HAL, est destinée au dépôt et à la diffusion de documents scientifiques de niveau recherche, publiés ou non, émanant des établissements d'enseignement et de recherche français ou étrangers, des laboratoires publics ou privés. 


\title{
All-optical signal processing based on self- induced polarization control in optical fibers
}

\author{
M. Guasoni, P. Y. Bony, M. Gilles, A. Picozzi, and J. Fatome \\ Laboratoire Interdisciplinaire Carnot de Bourgogne, \\ UMR 6303 CNRS - Université Bourgogne Franche-Comté, \\ 9 av. Alain Savary, 21078 Dijon, France
}

\begin{abstract}
In this contribution, we review our recent progress on the alloptical control of the state-of-polarization of light in optical fibers upon propagation in a system called Omnipolarizer. More precisely, in this device we exploit the unexpected capability of light to self-organize its own state-of-polarization, upon propagation in optical fibers, into universal and environmentally robust states. The underlying physical mechanism consists in a nonlinear cross-polarization feedback interaction between an arbitrary polarized incident signal and its own counterpropagating replica generated at the fiber end by means of a reflective element. Depending on the power ratio between the two waves, e.g. the reflective coefficient, this nonlinear selfrepolarization phenomenon offers a rich variety of dynamics for which we have highlighted three main working regimes identified by first a bistable operating regime, a polarization alignment process as well as a genuine chaotic behavior. We have fully characterized these three operating regimes with an excellent agreement between numerical and experimental results. Moreover, beyond the fundamental aspect of these first studies, we have then exploited this self-induced repolarization phenomenon in order to implement several proof-of-principles for all-optical signal processing. In particular, we have successfully demonstrated the spontaneous repolarization of a 10-Gbit/s Return-to-Zero optical signal without noticeable impairments. The bistability and associated hysteresis properties of the Omnipolarizer have been also exploited to implement an optical flip-flop memory as well as a 10-Gbit/s polarization-based data packet router. Finally, we have taken advantage of the chaotic dynamics of our device to demonstrate an all-optical scrambler enabling truly chaotic polarization diversity for $\mathbf{1 0}$ Gbit/s On/Off Keying wavelength division multiplexing applications.
\end{abstract}

\section{INTRODUCTION}

Despite outstanding technological developments in many fields of photonics, especially in all-fiber based systems, the state-of-polarization (SOP) of light remains the most elusive of all parameters, which is still challenging to predict and master. In fact, it is noteworthy that in the past decade tremendous and impressive progresses in the manufacturing of modern optical fibers have been realized. In particular, by implementing well-optimized spinning process during the drawing stage, fiber providers now propose standard Telecom fibers with spectacular weak levels of polarization-mode dispersion [1-4]. Nonetheless, the amount of residual birefringence combined with surrounding stress and variations such as bending, squeezing, vibrations or temperature fluctuations still make the polarization of light genuinely unpredictable after only a few hundreds of meters of propagation in fibers [5-10].

To overcome the impairments induced by this random nature of polarization in fiber-based systems, from a general point of view, the mitigation solutions implemented nowadays mainly rest upon combative strategies rather than on a preventive strategy. For instance, in current high-capacity coherent transmissions, polarization impairments such as polarization randomness, polarization-mode dispersion [1115], polarization depending loss [16] or cross-polarization interaction [17-18] are efficiently managed by means of digital signal processing implemented at the coherent receiver [1921].

Regarding highly polarization dependent systems such as on-chip integrated optical circuits or fiber-based nonlinear processing devices, special design and more or less complex polarization-diverse schemes (polarization diversity, bidirectional loop or polarization splitting/recombination) may ensure the mitigation of polarization dependent performances [22-25].

Another elegant strategy consists in mastering the SOP of light in order to prevent or mitigate some of these impairments. The most common and commercially available way is to implement an opto-electronic polarization tracking solution [26-30]. These devices generally consist in linear polarization transformations followed by partial diagnostic associated with an active feedback loop control driven by complex algorithms. Thanks to these techniques, records of polarization tracking or diversity speeds have been achieved, reaching several of $\mathrm{Mrad} / \mathrm{s}$. Nevertheless, these devices are essentially based on opto-electronic technologies, which could be seen as a limitation for future all-optical transparent networks. In this context and beyond its fundamental interest, the light-by-light polarization control represents a complementary and alternative approach. Indeed, the ability to all-optically master or trap the state-of-polarization of a light beam without polarization depending loss could encounter numerous applications in photonics. To this aim, several techniques have emerged in the literature in order to develop an "ideal polarizer" which could lead to a complete repolarization of an incident signal with $100 \%$ of efficiency, whilst preserving the quality of the temporal intensity profile. "Ideal" here meaning: 
without polarization depending losses in contrast to classical polarizers. As opposed to traditional polarizers which are known to vanish $50 \%$ of an unpolarized incoming signal, Heebner et al. first proposed in 2000 a "universal polarizer" performing repolarization of unpolarized light with high efficiency [31]. Subsequently, this phenomenon of polarization attraction or polarization pulling has been the subject of a growing interest in optical fiber based systems, involving the Raman effect [32-36], the stimulated Brillouin backscattering [37-38], the parametric amplification [39-40] as well as a counter-propagating four-wave mixing process, also called nonlinear cross-polarization interaction [41-55]. In particular, considering the counter-propagating interaction of two distinct optical beams injected at both ends of an optical fiber, it has been shown that an arbitrary polarized incident signal can be attracted toward a specific SOP, which is fixed by the SOP of the counter-propagating pump beam injected at the fiber output [44]. Several exploitations of this phenomenon of polarization attraction have been reported in the literature in conjunction with various types of optical functionalities, e.g., pulse reshaping [51], noise cleaner [52], data packet processing [53], Raman amplification [54], spatial mode attraction [55]. According to these works, the generally accepted point of view is that the injection of a pump beam at the fiber output is a prerequisite for the existence of the phenomenon of polarization attraction. The idea is that the fully polarized pump beam serves as a SOP reference for the signal beam, and thus plays the role of natural attractor for an incident unpolarized signal.

In opposition with this common belief, recent experimental results have unexpectedly demonstrated that a polarization attraction process can also occur in the absence of any SOP reference in a device called "Omnipolarizer" [56-57]. In this phenomenon of self-induced polarization attraction, the signal beam interacts with its own counter-propagating replica, which is generated at the fiber end by means of a reflected element, e.g., fiber Bragg-mirror, or amplified reflective fiber loop [56-58]. Furthermore, it has been shown that the Omnipolarizer may exhibit a chaotic polarization dynamics which could induce a fast and efficient polarization scrambling [64-70]. Both the attraction and the chaotic processes are two different aspects of the all-optical and selfinduced polarization control which takes place within the Omnipolarizer.

Our aim in this article is to provide a general overview of the dynamics and applications of this phenomenon of selfinduced polarization control, and to highlight the excellent agreement between theoretical, numerical and experimental results. In particular, after introducing the principle of operation and experimental implementation of the Omnipolarizer, we will report the existence and exploitation of 3 different working regimes, i.e. the bistability regime and the polarization alignment regime which have already been identified in refs. $[56,58]$ as well as a new scrambling regime. All of these peculiar regimes have then been exploited to provide several proof-of-principles for telecom applications including the repolarization of a 10-Gbit/s Return-to-Zero signal, optical memory and 10-Gbit/s data packet routing as well as a 10-Gbit/s wavelength-division-multiplexing (WDM) polarization scrambler. Finally, in the last section we trace out the conclusions.

\section{PRINCIPLE OF OPERATION}

As illustrated in Fig. 1, the Omnipolarizer basically consists in a few-km span of telecommunication fiber where a forward signal interacts through a Kerr nonlinear cross-polarization process with its own backward replica, which is generated by means of a back-reflection at the fiber end. The back-reflection is characterized by a reflection coefficient $\rho$ that defines the power-ratio between backward and forward waves. Practically, for standard telecom optical fibers of a few-km length, typical signal powers of $500 \mathrm{~mW}$ are then required to observe the present polarization dynamics. Two different setups have been implemented, which give rise to three different operational regimes depending on the reflection coefficient $\rho$.

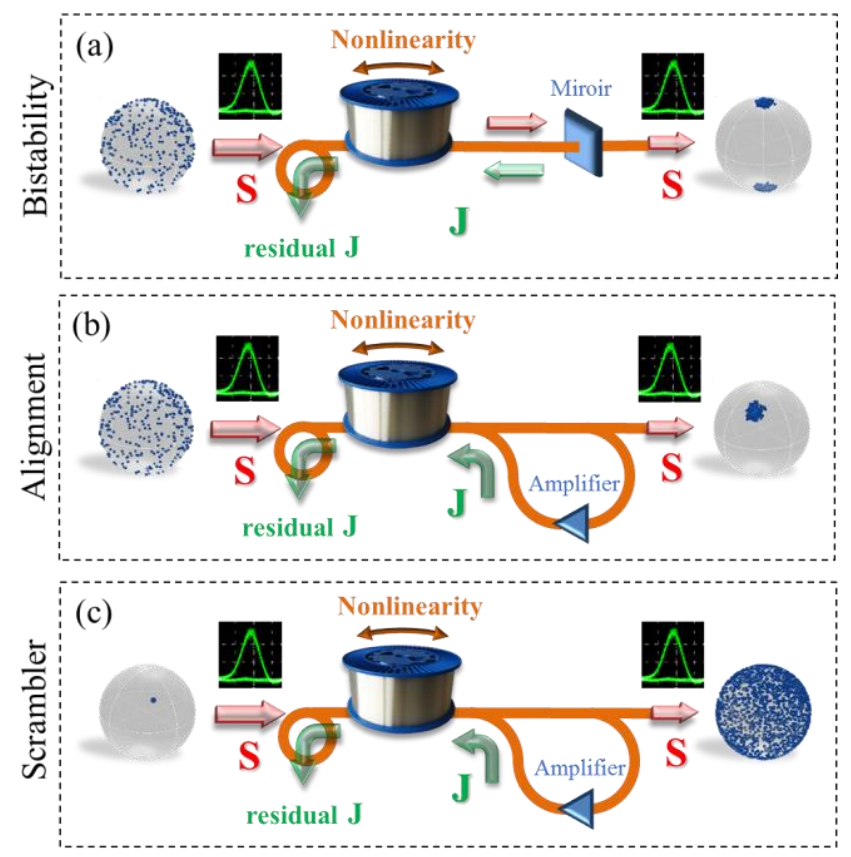

Fig. 1. Schematic setup of the Omnipolarizer and principle-of-operation of the self-induced polarization control. The left (right) Poincaré spheres illustrate the distribution of the input (output) SOP in the 3 identified regimes. In (a) the setup is passive: the backward beam $\mathbf{J}$ is produced by means of a reflective element (mirror). In (b,c) the setup is active: the backward beam is amplified in a reflective fiber loop. The eye diagrams illustrate the fact that the intensity profile is ideally preserved in all 3 the operating regimes, i.e. without polarization fluctuations transferred into the temporal domain.

In the first setup (Fig. 1a), called passive, the back-reflection is produced by means of a partially reflecting mirror, for which typically $0.8 \leq \rho \leq 1$. In this case, for any arbitrary polarized input signal, two fixed SOPs are produced that correspond to right or left circular states. The sign of the input signal ellipticity defines which of the two SOPs is obtained in output. Consequently, an initially depolarized signal, scrambled all over the Poincaré sphere (See Fig. 1a), is attracted towards 
both the poles of the sphere at the fiber end. Moreover, as we will see in the next sections, small polarization fluctuations of the input signal could make the output polarization to "jump" abruptly from the right to the left circular state, or vice versa, which is the main signature of a bistable regime. In the second configuration (Fig. 1b-c), called active, the backward signal is amplified in a reflective fiber loop basically made of an optical circulator and an amplifier. When the amplification is weak, typically $1.2 \leq \rho \leq 2$, for any arbitrary polarized input signal one single SOP is produced in output, whose position over the Poincaré sphere can be controlled by means of the polarization rotation imposed by the feedback loop. As a consequence, an initially depolarized signal is aligned towards one unique tunable SOP at the fiber end. We talk in this case of polarization alignment regime (Fig. 1b). On the other hand, a large amplification factor $\rho>>1$ leads to a chaotic polarization dynamics. Roughly speaking, this third regime is the opposite of the first two. Indeed in this case an initially fully polarized signal turns out to be completely depolarized in output, which corresponds to a scrambling regime (Fig. 1c).

\section{THEORETICAL DESCRIPTION}

In the following, we indicate with $\mathbf{S}=\left[\mathrm{S}_{1}, \mathrm{~S}_{2}, \mathrm{~S}_{3}\right]$ and $\mathbf{J}=\left[\mathrm{J}_{1}, \mathrm{~J}_{2}, \mathrm{~J}_{3}\right]$ the Stokes vectors for the forward and backward beams, respectively, whereas with $\mathbf{s}=\mathbf{S} /|\mathbf{S}|$ and $\mathbf{j}=\mathbf{J} /|\mathbf{J}|$ the normalized unitary Stokes vectors that represent the SOP. The evolution of $\mathbf{S}$ and $\mathbf{J}$ in the fiber is governed by the following coupled equations [56]:

$$
\begin{aligned}
& c^{-1} \partial_{t} \mathbf{S}+\partial_{z} \mathbf{S}=\mathbf{S} \times \mathrm{D} \mathbf{J}-a \mathbf{S} \\
& c^{-1} \partial_{t} \mathbf{J}-\partial_{z} \mathbf{J}=\mathbf{J} \times \mathbf{D} \mathbf{S}-\alpha \mathbf{J}
\end{aligned}
$$

where $\mathrm{D}=\gamma \cdot \operatorname{diag}(-8 / 9,-8 / 9,8 / 9)$ is a diagonal matrix, $\gamma$ and $\alpha$ are the nonlinear Kerr coefficient and the propagation losses of the fiber, respectively, and $c$ is the speed of light in the fiber. According to this notation, the component $\mathrm{s}_{3}$ indicates the forward signal ellipticity. The boundary condition at the fiber end reads as $\mathbf{J}(\mathrm{z}=\mathrm{L}, \mathrm{t})=\rho \mathrm{RS}(\mathrm{z}=\mathrm{L}, \mathrm{t})$, where $\mathrm{R}$ is a $3 \times 3$ rotation matrix modeling the polarization rotation in the reflectiveloop, whereas $\mathrm{L}$ indicates the fiber length and $\rho=|\mathbf{J}(\mathrm{z}=\mathrm{L}, \mathrm{t})|$ / $|\mathbf{S}(\mathrm{z}=\mathrm{L}, \mathrm{t})|$. It is important to notice that chromatic dispersion is here neglected in our model since it does not impact significantly the efficiency of the cross-polarization interaction due to the counter-propagating nature of the phenomenon. Furthermore, considering the incident signals involved in our experiments, their corresponding dispersive lengths appear much longer than the segment of fiber implemented within the Omnipolarizer. Despite their simplicity, Eqs. (1) capture all the essential properties of the polarization dynamics in the Omnipolarizer, which is demonstrated by the excellent agreement with experimental results discussed in the next sections. Moreover, Eqs. (1) are formulated in terms of Stokes vectors, which are preferable to the original amplitudes of the electric field as they represent the more natural and convenient way to analyze polarization phenomena in the fiber.
An important property of the system under analysis, which immediately comes out from Eqs. (1) is that the intensity profiles $|\mathbf{S}|$ and $|\mathbf{J}|$ are preserved except for propagation losses and a temporal shift (as illustrated by the eye-diagrams in Fig. $1)$, namely $|\mathbf{S}(\mathrm{z}, \mathrm{t})|=|\mathbf{S}(0, \mathrm{t}-\mathrm{z} / \mathrm{c})| \exp (-\alpha \mathrm{z})$ and $|\mathbf{J}(\mathrm{z}, \mathrm{t})|=$ $|\mathbf{J}(0, t+z / c)| \exp (\alpha z-\alpha \mathrm{L})$. This prevents input polarization fluctuations to be transferred into the temporal domain as large output intensity variations, which is typical of linear polarizers or repolarization methods based on a polarization depending gain process.

The system dynamics in the 3 regimes previously outlined is closely related to the stability of the stationary states, which are the solutions of Eqs. (1) when dropping the time derivatives. When the powers of the forward and backward beams are similar, which is the case for the first two regimes, only the stable stationary SOPs, here indicated with sstat and $\mathbf{j}$ stat, can play the role of polarization attractors for the output forward and backward signals. As a rule of thumb, it was found that stable stationary states are characterized by a nonoscillatory behavior, while non-stable states are oscillating along the fiber length [49] (see Fig. 2).
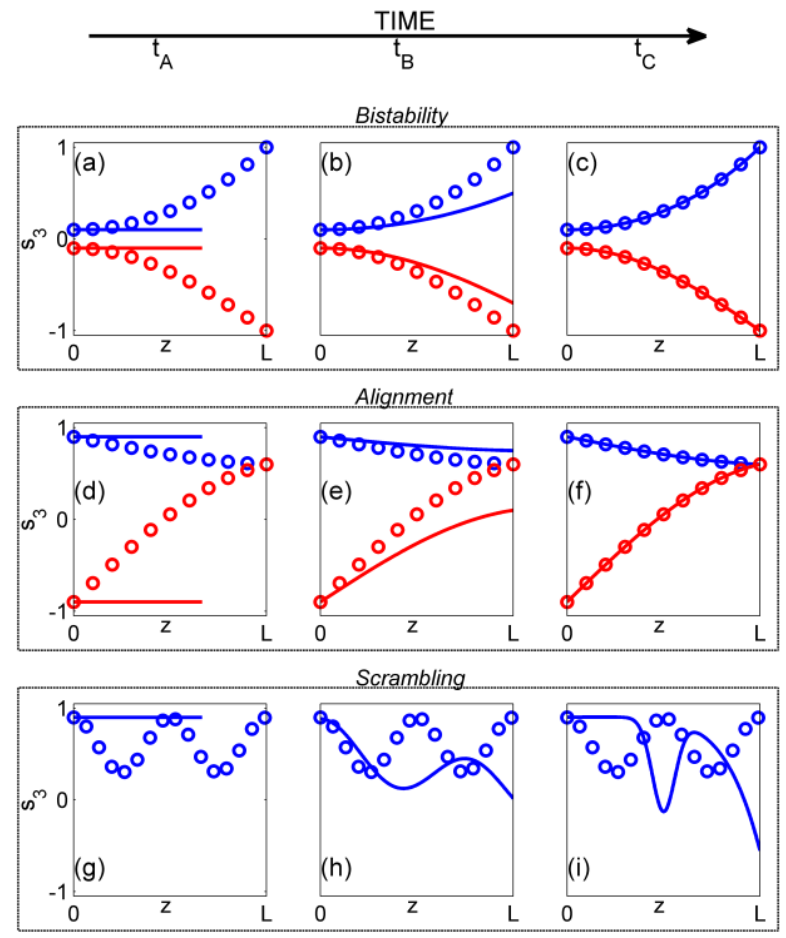

Fig. 2. Spatial evolution along the fiber length of the normalized Stokes component $\mathrm{s}_{3}$ (solid lines) in the 3 operational regimes under analysis. Three consecutive instants are represented, which are $t_{\mathrm{A}}<\mathrm{L} / \mathrm{c}$ (left panels a,d,g); $t_{\mathrm{B}}>\mathrm{L} / \mathrm{c}$ (central panels $\mathrm{b}, \mathrm{e}, \mathrm{h}$ ); $\mathrm{t}_{\mathrm{C}}>>\mathrm{L} / \mathrm{c}$ (rigth panels $\mathrm{c}, \mathrm{f}, \mathrm{i}$ ).Corresponding stationary solutions $\mathrm{s}_{3, \text { stat }}$ are represented in circles. Panels a-c: bistable regime. Two cases are represented that correspond to two different input polarizations $\mathrm{s}_{3}(\mathrm{z}=0)=+0.1$ (blue lines and circles) and $\mathrm{s}_{3}(\mathrm{z}=0)=-0.1$ (red lines and circles). In both cases, despite the slight difference in input, $s_{3}$ is asymptotically attracted in time towards the corresponding stable stationary solution $\mathrm{s}_{3, \text { stat }}$. Panels d-f: alignment regime. Two cases are represented that correspond to two different input polarizations $\mathrm{s}_{3}(\mathrm{z}=0)=0.9$ (blue lines and circles) and $\mathrm{s}_{3}(\mathrm{z}=0)=-0.9$ (red lines and circles). In both cases $\mathrm{s}_{3}$ is asymptotically attracted in time towards a unique stable stationary solution $\mathrm{s}_{3, \text { stat }}$. Panels g-i: scrambling regime. The stationary solution is unstable and consequently $s_{3}$ does not converge towards $\mathrm{s}_{3, \text { stat }}$ but fluctuates in time without reaching a fixed state. 
Figures 2a-c display a schematic illustration of the bistable process discussed above $(0.8 \leq \rho \leq 1)$. The component $\mathrm{s}_{3}$ of $\mathbf{s}$ is depicted along the fiber (solid line) at 3 consecutive times $t_{A}<L / c, t_{B}>L / c$ and $t_{C}>>L / c$, where $L / c$ defines the end-to-end propagation time in the fiber. For the sake of simplicity, we initially assume that the input signal is constant in time, i.e. $\mathbf{s}(\mathrm{z}=0, \mathrm{t}) \equiv \mathbf{s}(\mathrm{z}=0)$.

At $t_{A}<L / c$ (panel a) the backward replica has not been generated, yet, therefore $\mathbf{S}$ is unaffected by nonlinear coupling with $\mathbf{J}$ and propagates unchanged in the fiber. On the other hand, at $t_{B}>L / c$ (panel $b$ ), $\mathbf{J}$ has been generated and nonlinearly interacts with $\mathbf{S}$, so that $\mathbf{S}$ gradually converges towards the stable state sstat (depicted with circles). The attraction is stronger and stronger with time, so that at $\mathrm{t}_{\mathrm{C}}>>\mathrm{L} / \mathrm{c} \mathbf{s}\left(\mathrm{z}, \mathrm{t}=\mathrm{t}_{\mathrm{C}}\right)$ and $\mathbf{s}_{\text {stat }}(\mathrm{z})$ practically coincide $\left(\mathrm{s}_{3}\left(\mathrm{z}, \mathrm{t}=\mathrm{t}_{\mathrm{C}}\right) \simeq \mathrm{s}_{3, \text { stat }}(\mathrm{z})\right.$ in panel c).

As we will see in next section, there is a unique stable stationary state associated with a given value $\mathbf{s}_{\text {stat }}(\mathrm{z}=0)$, therefore $\mathbf{s}(\mathrm{z}, \mathrm{t})$ converges towards the stable stationary state such that $\mathbf{S}_{\mathrm{stat}}(\mathrm{z}=0)=\mathbf{s}(\mathrm{z}=0)$. Furthermore, the stable stationary state basically depends on the reflection coefficient $\rho$ and on the total number $\mathrm{N}=\mathrm{L} / \mathrm{L}_{\mathrm{NL}}$ of nonlinear lengths, being $\mathrm{L}_{\mathrm{NL}}=(\gamma|\mathbf{S}|)^{-1}$ the characteristic nonlinear length [71]. One peculiar property of the system under analysis (see Section $\mathrm{V}$ ) is that if $\rho \simeq 1$ and $N \gg>1$ (typically $N \geq 4$ ), which is the case in highly nonlinear regime, then $\mathrm{s}_{3, \text { stat }}(\mathrm{z}=\mathrm{L}) \simeq \operatorname{sign}\left[\mathrm{s}_{3, \text { stat }}(\mathrm{z}=0)\right]$. For typical involved fibers which are few-km long and whose Kerr coefficient is $\gamma \approx 2 \mathrm{~W}^{-1} \mathrm{~km}^{-1}$, a signal power in the order of $500 \mathrm{~mW}$ is required to accomplish the aforementioned highlynonlinear regime. The Omnipolarizer is then bistable and characterized by 2 pools of attraction. That is to say: the output value $s_{3, \text { stat }}(\mathrm{z}=\mathrm{L})$ gets close to +1 or -1 simply depending on the sign of $s_{3, \text { stat }}(\mathrm{z}=0)$. Furthermore, a slight different input condition of $s_{3}(\mathrm{z}=0)$ may give rise to a completely opposite output polarization, which is the case displayed in Fig. 2c, where if $\mathrm{s}_{3}(\mathrm{z}=0)=+0.1$ then $\mathrm{s}_{3}\left(\mathrm{z}=\mathrm{L}, \mathrm{t}=\mathrm{t}_{\mathrm{C}}\right) \simeq \mathrm{s}_{3, \mathrm{stat}}(\mathrm{z}=\mathrm{L}) \simeq+1$, otherwise if $\mathrm{s}_{3}(\mathrm{z}=0)=-0.1$ then $\mathrm{s}_{3}\left(\mathrm{z}=\mathrm{L}, \mathrm{t}=\mathrm{t}_{\mathrm{C}}\right) \simeq \mathrm{s}_{3, \text { stat }}(\mathrm{z}=\mathrm{L}) \simeq-1$.

Similarly to the bistable regime, also in the alignment regime $(1.2 \leq \rho \leq 2)$ there is an asymptotic convergence of $\mathbf{s}$ towards the stable stationary state $\mathbf{S}$ stat such that $\mathbf{S s t a t}(\mathrm{z}=0)=$ $\mathbf{s}(\mathrm{z}=0)$. However, as we will clarify in Section VI, in this case the output $\mathbf{S}_{\text {stat }}(\mathrm{Z}=\mathrm{L})$ is almost independent of the input $\mathbf{s}_{\text {stat }}(\mathrm{z}=0)$ and thus of $\mathbf{s}(\mathrm{z}=0)$. For this reason we observe a unique attraction SOP at the fiber output, which is the case displayed in Fig. 2d-f, where $\mathrm{s}_{3}\left(\mathrm{z}=\mathrm{L}, \mathrm{t}=\mathrm{t}_{\mathrm{C}}\right) \simeq \mathrm{s}_{3, \text { stat }}(\mathrm{z}=\mathrm{L})=+0.5$ whatever the input value $s_{3}(z=0)$ is. Note that the value $\mathrm{s}_{3, \text { stat }}(\mathrm{z}=\mathrm{L})=+0.5$ in this example is purely indicative: actually, as we will see in Section VI, Sstat $(\mathrm{z}=\mathrm{L})$ is tunable all over the Poincare sphere by means of the rotation R.

Contrary to the bistable and the alignment regime, the scrambling regime $(\rho>>1)$ is characterized by a large power imbalance between the forward and the backward beam. This makes the stationary states oscillating with a spatial period inferior to the fiber length, so that they become unstable and cannot play the role of attractors anymore. As a consequence,
$\mathbf{S}$ does not converge towards Sstat in time, but varies without reaching a fixed state. Whatever the input polarization is, the output polarization $\mathbf{s}(\mathrm{z}=\mathrm{L}, \mathrm{t})$ varies endlessly in time and becomes therefore temporally scrambled (Fig. $2 \mathrm{~g}$-i). As we will see in Section VII the time scale of the output polarization fluctuations can be controlled by means of the coefficient $\rho$, which leads to the implementation of an efficient all-optical polarization scrambler.

\section{EXPERIMENTAL SETUP}

Figure 3 depicts the general experimental implementation especially designed to characterize the 3 different operating regimes of the Omnipolarizer. Depending on the working regime and in fine optical functionality, the setup can combine several kinds of input signals and characterization tools. First of all, the Omnipolarizer basically consists in a single span of non-zero dispersion shifted fiber (NZ-DSF) of a few kilometers encapsulated between a reflected element at the output side and an optical circulator at the input to evacuate the residual backward signal. The fiber is selected with a normal dispersion at the signal wavelength so as to avoid any modulational instability or soliton compression effects. The polarization mode dispersion (PMD) coefficient of the fiber is also ideally chosen to be below $0.05 \mathrm{ps} / \mathrm{km}^{1 / 2}$ so as to keep a low level of residual birefringence which could decrease the efficiency of the polarization control. The input signal is amplified by means of an Erbium doped fiber amplifier (EDFA) before injection into the fiber under-test. Moreover, considering the power levels involved in our experiments, typically half a Watt in average, a few kilometers of fiber is enough to propagate the signal upon several normalized nonlinear lengths and thus to ensure an efficient repolarization process.

In the passive configuration of the Omnipolarizer (see (a) in the setup scheme), the reflected element which produces the feedback signal is typically a fiber Bragg grating (FBG) but could be also a metallic coating or a mirror. The present integrated FBG is characterized by a bandwidth of $1 \mathrm{THz}$ centered on $1550 \mathrm{~nm}$ and a reflective coefficient of $97 \%$. Therefore, $3 \%$ of the transmitted signal actually corresponds to the output processed signal. In its active configuration (path (b) in the setup), the reflective element consists in a fiber loop setup made of an optical circulator, an additional EDFA, a polarization controller adjusting the rotation matrix $\mathrm{R}$, as well as a 90:10 tap coupler to evacuate the resulting output signal for analysis. In this configuration, the reflective coefficient $\rho$ can be obviously larger than 1 and is thus directly related to the gain of this second EDFA.

For fundamental characterizations of the Omnipolarizer, the initial signal consists in a polarized $100-\mathrm{GHz}$ bandwidth, partially incoherent wave centered on $1550 \mathrm{~nm}$ obtained by spectral filtering of an Erbium-based spontaneous noise emission source (ASE). This spectral bandwidth, rather than a pure continuous wave, is used here to avoid any impairment due to the stimulated Brillouin backscattering in the optical fiber of the Omnipolarizer. Indeed, since the Omnipolarizer is 
based on a strong nonlinear interaction between counterpropagating waves, the device is first clearly limited by Brillouin back-scattering when using pure CWs. Consequently, Brillouin suppression methods have to be implemented to use CW signals such as phase modulation, strain or temperature distribution along the fiber length as well as aluminum doped core fibers [59-63]. For Telecom processing demonstrations, the signal under-test consists in a 10-Gbit/s On/Off Keying (OOK) Return-to-Zero (RZ) signal centered on $1555 \mathrm{~nm}$. The 10-Gbit/s RZ signal is generated from a $10-\mathrm{GHz}$ mode-locked fiber laser delivering 2.5-ps pulses at $1555 \mathrm{~nm}$. A programmable optical filter is used to temporally stretch the initial pulses to $25 \mathrm{ps}$ which are then intensity modulated by means of a $2^{7}-1$ pseudo-random bit sequence (PRBS). Note that this short length of the PRBS sequence undertest is here only used to provide a first proofof-principle of the polarization switching concept. Nevertheless, we expect that similar performances would have been obtained with much longer sequences. Moreover, since the polarization attraction process has already been demonstrated for RZ or Return-to-Zero modulation formats
[43], we expect that neither the pulse width nor the OOK format would impact the performance of our device. Nevertheless, we expect that a limitation of the device could be observed when short data-packets would be injected into the Omnipolarizer instead of continuous data flows. Indeed, for packets duration below the time-response, the repolarization effect would be affected. However, it has been shown in ref. [53] that packets of $205 \mathrm{~ns}$ time-duration have been efficiently repolarized by means of a counterpropagating configuration of the polarization attractor so we expect that same duration could be efficiently routed in the present device. Before injecting the incident signal into the device, an opto-electronic polarization controller/scrambler (PS) enables us to alternatively scramble (at a rate of $0.5 \mathrm{kHz}$ ) or create a specific polarization trajectory on the Poincaré sphere. At the output of the Omnipolarizer, the resulting signal is filtered (F-3) to suppress the spontaneous noise outside the signal bandwidth and is finally characterized both in the polarization and in the temporal domains by means of a polarimeter and a 50-GHz bandwidth sampling oscilloscope for Telecom purpose, respectively.
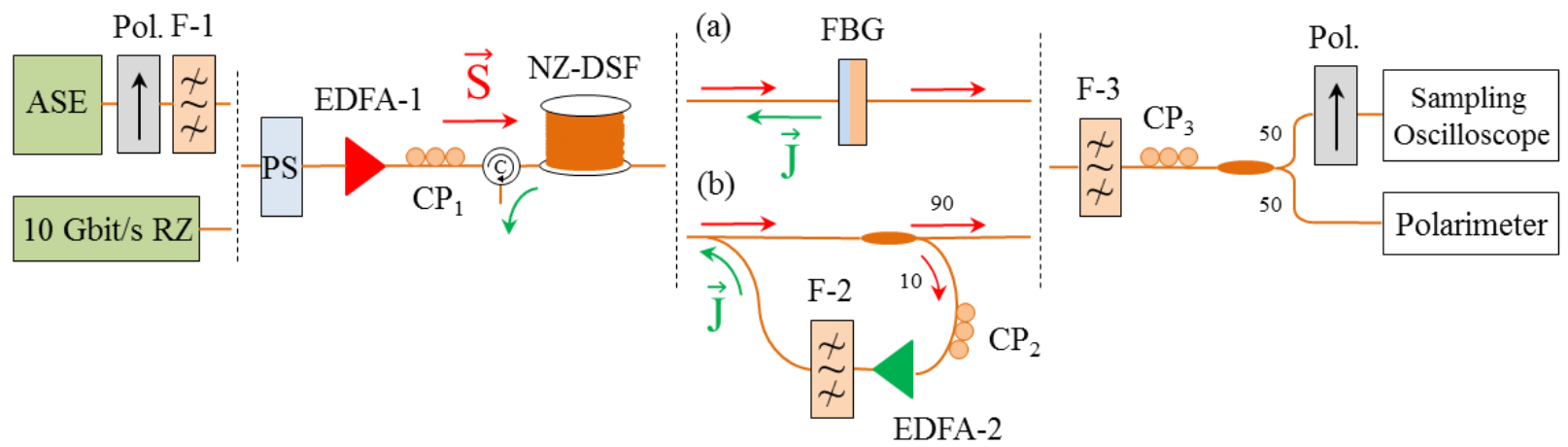

Fig. 3. Experimental setup. (a) Passive configuration of the Omnipolarizer (b) Active implementation. ASE: amplified spontaneous noise emission, Pol: inline polarizer, PS: polarization scrambler, EDFA: Erbium doped fiber amplifier, NZ-DSF: non-zero dispersion fiber, FBG: fiber Bragg grating, PC: polarization controller, F: optical filter.

\section{BISTABILITY: FROM HYSTERESIS TO FLIP-FLOP MEMORIES AND SWITCHING OPERATIONS}

In this section we gain a deeper physical understanding of the bistable process outlined in Section III and we discuss the experimental proof-of-principle of some useful applications based on polarization bistability [58].

The stationary system associated to Eqs. (1) when losses are neglected admits as a constant of motion the vector $\mathbf{K}=\mathbf{S}$-DJ. By insertion of DJ $=\mathbf{S}-\mathbf{K}$ in the stationary system we obtain the equality $\partial_{z} \mathbf{S}=\mathbf{K} \times \mathbf{S}$, whose solution for $\mathbf{S}$ are rotations around $\mathbf{K}$ corresponding to circles of spatial period $2 \pi /|\mathbf{K}|[56]$.

Taking into account that in the passive setup $\rho \simeq 1$ and there is no rotation imposed on $\mathbf{J}$ at the fiber end, i.e. $\mathrm{R}=\operatorname{diag}(1,1,1)$, then the boundary condition associated to the stationary system in $\mathrm{z}=\mathrm{L}$ simply reads as $\mathbf{J}(\mathrm{z}=\mathrm{L})=\mathbf{S}(\mathrm{z}=\mathrm{L})$.

Consequently, the component $\mathrm{K}_{3}$ of $\mathbf{K}$ is null and therefore the system $\partial_{z} \mathbf{S}=\mathbf{K} \times \mathbf{S}$ is symmetric with respect to rotations around the $S_{3}$ direction. This practically allows restricting the study to find the relation between the input ellipticity $s_{3}(z=0)$ and the output ellipticity $\mathrm{s}_{3}(\mathrm{z}=\mathrm{L})$ of the stationary states. This curve is displayed in Fig. 4a-b for different values of the number $\mathrm{N}$ of nonlinear lengths. If $\mathrm{N} \simeq 0$ (Fig. $4 \mathrm{a}$ ) we clearly get a quasistraight line that corresponds to the limit $\mathrm{L} \simeq 0$, i.e. $\mathrm{s}_{3}(\mathrm{z}=\mathrm{L}) \simeq$ $\mathrm{s}_{3}(\mathrm{z}=0)$. On the other hand, the more $\mathrm{N}$ increases, the more the curve is deformed until it exhibits a vertical tangent when $\mathrm{N}=\pi / 2$. The curve becomes multivalued for $\mathrm{N}>\pi / 2$ (Fig. $4 \mathrm{~b}$ ), so that for a given input $s_{3}(z=0)$ we may find several outputs $\mathrm{s}_{3}(\mathrm{z}=\mathrm{L})$, corresponding to a variety of stationary solutions $\mathrm{s}_{3}(\mathrm{z})$ associated with $\mathrm{s}_{3}(\mathrm{z}=0)$.

However, only one among these stationary solutions is stable. Indeed, if $-1 \leq \mathrm{s}_{3}(\mathrm{z}=0) \leq 0$ then only the lower-valued solution $s_{3}(z=L)$, represented by the blue solid line in Fig. $4 b$, is the only one related to a stationary state which is not oscillating along the fiber length, and is thus stable [49]. Conversely, if $0 \leq \mathrm{s}_{3}(\mathrm{z}=0) \leq 1$ then only the higher-valued solution $\mathrm{s}_{3}(\mathrm{z}=\mathrm{L})$, represented by the red solid line in Fig. 4b, is the one related to a stable stationary state and plays the role of polarization attractor. 

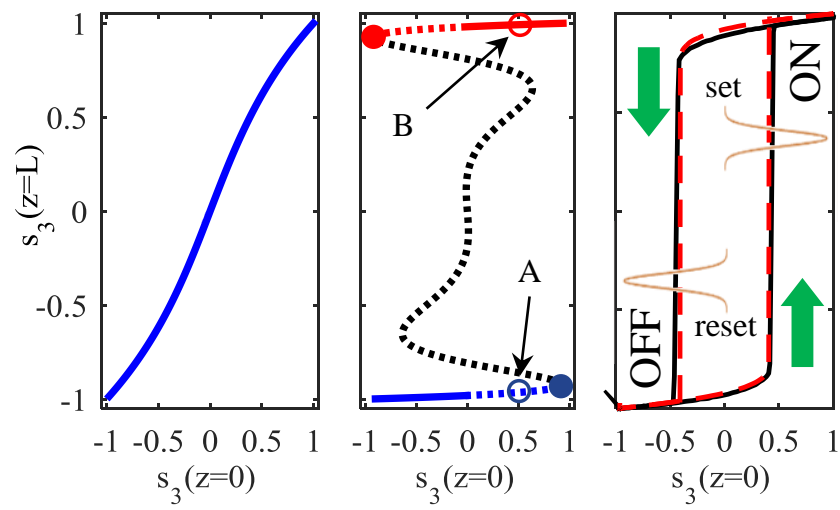

Fig. 4. (a): $s_{3}(z=0)$ versus $s_{3}(z=L)$ for the stationary solutions of Eq.(1) in the passive setup $\rho=1, R=\operatorname{diag}([1,1,1])$, null losses and $N=0.4$. All points of the curve correspond to stable stationary solutions. (b): same as in (a) but when $\mathrm{N}=4$. Solid blue and red lines correspond to the stable states such that $\mathrm{s}_{3}(\mathrm{z}=\mathrm{L}) \simeq$ 1 and $s_{3}(\mathrm{z}=\mathrm{L}) \simeq+1$, respectively. Dashed blue and red lines correspond to the metastable states. The black dashed line corresponds to the set of unstable states. The blue and red spots in $\mathrm{s}_{3}(\mathrm{z}=0)= \pm 0.85$ identify the extremities of the line of unstable states. The empty circles A and B represent respectively the metastable and the stable states corresponding to $s_{3}(z=0)=0.5$. (c): Hysteresis cycle recorded experimentally at the output of the Omnipolarizer (solid black line). The input signal consists in a $100-\mathrm{GHz}$ polarized incoherent signal with an average power of $570 \mathrm{~mW}(\rho=0.9)$. The component $\mathrm{s}_{3}(\mathrm{z}=0)$ varies in time from -1 to +1 and vice-versa with a ramping time of $200 \mathrm{~ms}$. Numerical solution of Eqs.(1) are also represented (dashed red line)

Furthermore, it is clear from Fig. $4 \mathrm{~b}$ that when $\mathrm{N} \gg>1$ then the blue and red segments correspond respectively to $s_{3}(\mathrm{z}=\mathrm{L}) \simeq-1$ and $\mathrm{s}_{3}(\mathrm{z}=\mathrm{L}) \simeq+1$. Therefore, as previously anticipated in Section III, in a highly nonlinear regime the stable stationary states are such that $\mathrm{s}_{3}(\mathrm{z}=\mathrm{L}) \simeq \operatorname{sign}\left[\mathrm{s}_{3}(\mathrm{z}=0)\right]$ [56]. Consequently, if a constant-in-time input SOP $\mathbf{s}(\mathrm{z}=0, \mathrm{t}) \equiv \mathbf{S}(\mathrm{z}=0)$ that is in the north (south) hemisphere of the Poincaré sphere is injected into the fiber, namely $\mathrm{s}_{3}(\mathrm{z}=0)>0\left(\mathrm{~s}_{3}(\mathrm{z}=0)<0\right)$, then the corresponding output $\mathbf{s}(\mathrm{z}=\mathrm{L}, \mathrm{t})$ will relax towards the north (south) pole of the sphere, that is $\mathrm{s}_{3}(\mathrm{z}=\mathrm{L}, \mathrm{t}) \simeq+1\left(\mathrm{~s}_{3}(\mathrm{z}=\mathrm{L}, \mathrm{t}) \simeq-1\right)$.

One may wonder what happens if the input $\mathbf{s}(\mathrm{z}=0, \mathrm{t})$ is not constant but varies in time. On the basis of what explained above, we may be wrongly induced to think that if $s_{3}(z=0, t)$ moves from negative values to positive values (i.e. it changes sign) then the corresponding output $\mathrm{s}_{3}(\mathrm{z}=\mathrm{L}, \mathrm{t})$ suddenly jumps from -1 to +1 . This is not true because another class of stationary solutions exists that are metastable, and are indicated by the blue and red dashed lines in Fig. 4b. Roughly speaking, these metastable states are stable under small perturbations and can be adiabatically reached from the stable states. This means that if we slowly vary $\mathrm{s}_{3}(\mathrm{z}=0, \mathrm{t})$ from -1 towards +1 , after having covered the blue solid line of stable states in Fig. $4 \mathrm{~b}$ we enter in the blue dashed line of metastable states which starts in correspondence of $s_{3}(z=0)=0$. Moving further, we find the first vertical tangent, which is indicated by the blue spot in Fig. $4 \mathrm{~b}$ and that corresponds to the first unstable state at $\mathrm{s}_{3}(\mathrm{z}=0)=0.85$. It is for an input $0 \leq \mathrm{s}_{3}(\mathrm{z}=0) \leq 0.85$ that the output $\mathrm{s}_{3}(\mathrm{z}=\mathrm{L}, \mathrm{t})$ abruptly jumps towards the opposite branch of stable states, that is the red solid line in Fig. 4b. The switching value $\mathrm{s}_{3}(\mathrm{z}=0)$ strictly depends on how quickly the input $\mathrm{s}_{3}(\mathrm{z}=0, \mathrm{t})$ varies: the more its transition from -1 to +1 is slow, the larger is the switching value, but in any case the upper limit is represented by the value corresponding to the first unstable state, which is $\mathrm{s} 3(\mathrm{z}=0)=0.85$ in the case under analysis. As an example, in Fig. $3 \mathrm{~b}$ the switching value $\mathrm{s}_{3}(\mathrm{z}=0)=0.5$ gives rise to the jump of $\mathrm{s}_{3}(\mathrm{z}=\mathrm{L}, \mathrm{t})$ from the point $\mathrm{A}$, which is close to -1 and is related to a metastable state, to the point $\mathrm{B}$, which is close to +1 and corresponds to a stable stationary state.

Clearly, the same could be said when $s_{3}(z=0, t)$ moves from +1 towards -1 . In this case, after having covered the red solid line of stable states, we enter in the red dashed line of metastable state and we find the vertical tangent indicated by the red spot in correspondence of $\mathrm{s}_{3}(\mathrm{z}=0, \mathrm{t})=-0.85$. For a switching value $-0.85 \leq \mathrm{s}_{3}(\mathrm{z}=0) \leq 0$ the output $\mathrm{s}_{3}(\mathrm{z}=\mathrm{L}, \mathrm{t})$ suddenly jumps towards the opposite branch of stable states, that is the blue solid line in Fig. 4b.

These abrupt transitions between the upper and the lower branches of the curve in Fig. $4 \mathrm{~b}$ are a clear signature of a hysteresis phenomenon. This behavior, here described in the ideal case of zero losses and unitary reflection coefficient, is however still observed for typical weak propagation losses of $0.2 \mathrm{~dB} / \mathrm{km}$ and for $0.8 \leq \rho \leq 1$. Figure $3 \mathrm{c}$ illustrates a complete hysteresis cycle obtained in experiments when employing a 4$\mathrm{km}$ long non-zero dispersion shifted fiber (NZ-DSF) characterized by a chromatic dispersion of $-1.16 \mathrm{ps} / \mathrm{nm} / \mathrm{km}$, a Kerr coefficient $\gamma=1.7 \mathrm{~W}^{-1} \mathrm{~km}^{-1}$ and a PMD coefficient of 0.05 $\mathrm{ps} / \mathrm{km}^{1 / 2}$. The reflection coefficient $\rho \simeq 0.9$ and propagation losses are $0.2 \mathrm{~dB} / \mathrm{km}$. We have carefully generated on the polarized $100-\mathrm{GHz}$ incoherent wave an adiabatic transition making the input $\mathrm{s}_{3}(\mathrm{z}=0, \mathrm{t})$ to vary from -1 to +1 and vice-versa with a ramping time of $200 \mathrm{~ms}$ thanks to the opto-electronic input polarization controller. The input average power is 570 $\mathrm{mW}(27.5 \mathrm{dBm})$ and the corresponding nonlinear length is $\mathrm{L}_{\mathrm{NL}}=1.03 \mathrm{~km}$, so that $\mathrm{N} \simeq 4$. The switching of the output $\mathrm{s}_{3}(\mathrm{z}=\mathrm{L}, \mathrm{t})$ between the two branches of the hysteresis when the input $\mathrm{s}_{3}(\mathrm{z}=0, \mathrm{t}) \simeq \pm 0.4$ is clearly visible. Note that the output $\mathrm{s}_{3}$ component maintains its value close to -1 (OFF state in Fig. 4c) or +1 (ON state in Fig. 4c) and that transitions are remarkably sharp, so that the hysteresis cycle is well opened. As previously underlined, this is due to the presence of a strong nonlinearity $(\mathrm{N} \simeq 4)$. Note also the excellent agreement with numerical results, obtained by solution of Eqs. (1) including exact experimental parameters. This confirms the full validity of Eqs. (1) for the description of the polarization dynamics in the Omnipolarizer.

An interesting application of the hysteresis loop is the possibility to imprint or reset an ON or OFF state on the system and to maintain this state even if the flipping cause has disappeared, in such a way to implement a polarization-based flip-flop memory [58]. The principle of operation can be inferred by Fig. 4c. When a set pulse on the $\mathrm{s}_{3}$ component is injected into the fiber, its peak value switches the output $s_{3}$ component to the $\mathrm{ON}$ state close to +1 . Afterward, the input set pulse vanishes to 0 but the system has stored the ON state because of the hysteresis properties. It is only by means of a reset pulse that the output $\mathrm{s}_{3}$ component drops back to the OFF state close to -1 so as to clear the memory and maintains this condition until a next set pulse is applied.

Figure 5a-b illustrates the experimental proof-of-principle 
observation of a polarization-based flip-flop memory that is loaded (cleared) by a train of set (reset) pulses, respectively. The fiber and the average input power are those employed when reproducing the hysteresis cycle of Fig. 4c. Therefore, the peaks of the set and reset input pulses should be respectively larger than +0.4 and lower than -0.4 in order to cause the transition of the output $\mathrm{s}_{3}$ from the OFF to the ON state and vice-versa.

A random sequence of set/reset input spikes $s_{3}(z=0, t)$ with width of $2 \mathrm{~ms}$, a rise time of $20 \mu \mathrm{s}$ and peaks of \pm 0.7 was imprinted on the $100-\mathrm{GHz}$ polarized incoherent wave by means of the input opto-electronic polarization controller. This signal was then amplified to an average power of $570 \mathrm{~mW}$. The $\mathrm{s}_{3}$ input sequence as a function of time can be monitored in Fig. 5a. When injected into the Omnipolarizer, this set/reset polarization spike sequence gives rise to the output $\mathrm{s}_{3}(\mathrm{z}=\mathrm{L}, \mathrm{t})$ displayed in Fig. 5b. We can clearly observe that the system is able to perpetually store the OFF/ON state until a new input set/reset control pulse appears, thus demonstrating the storage capacity of this polarization-based flip-flop memory. We stress that the sharp edges of the output wave $s_{3}(\mathrm{z}=\mathrm{L}, \mathrm{t})$ represent the temporal counterpart of the sharp transitions in the hysteresis cycle of Fig. 4c, and are thus linked to the strong system nonlinearity: as a rule of thumb the larger is $\mathrm{N}$, the sharper are the edges.
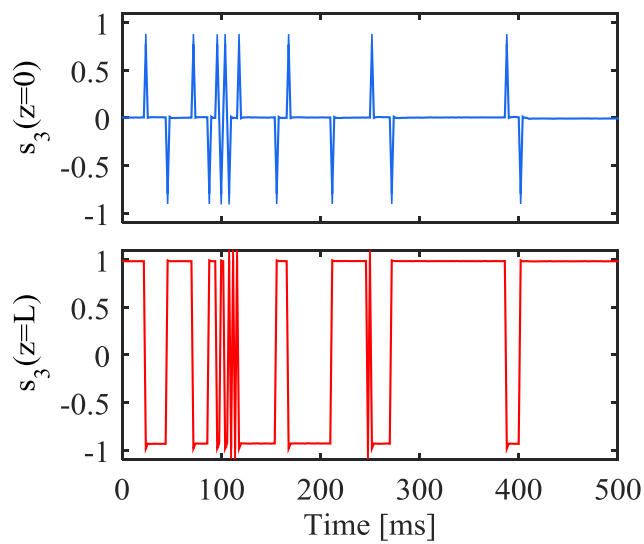

Fig. 5. Experimental proof-of-principle of a flip-flop memory based on the hysteresis process outlined in Fig.3c. The Omnipolarizer consists in a 4-km long NZ-DSF fiber with a 100-GHz polarized incoherent input signal with an average power of $570 \mathrm{~mW}$ and $\rho=0.9$ (a): Triggering set/reset $\mathrm{s}_{3}(\mathrm{z}=0, \mathrm{t})$ sequence

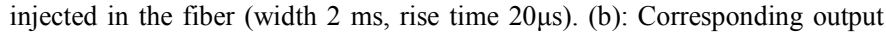
$\mathrm{s}_{3}(\mathrm{z}=\mathrm{L}, \mathrm{t})$.

Another interesting exploitation of the hysteresis properties of the system may consist in the implementation of all-optical polarization switching operation [58]. The key-principle is the same as the flip-flop memory previously shown, that is the sharp transitions in presence of set/reset input events. To this aim, the initial partially incoherent signal is now replaced by a 10-Gbit/s RZ signal. As in the flip-flop memory experiment reported above, an arbitrary sequence of 2-ms set/reset s 3 spikes is finally imprinted on the incident $10-\mathrm{Gbit} / \mathrm{s}$ signal thanks to the opto-electronic polarization controller. In order to characterize the switching efficiency, a polarization beam splitter (PBS) is inserted at the output of the Omnipolarizer to monitor the intensity profile on both orthogonal axes. The switching packet envelopes are first detected with a lowbandwidth oscilloscope while the 10-Gbit/s eye-diagrams are monitored by means of a $50-\mathrm{GHz}$ bandwidth sampling oscilloscope. Basically, the first axis of the PBS detects the magnitude $\left|\mathrm{s}_{3}(\mathrm{z}=\mathrm{L}, \mathrm{t})\right|$, whereas the second axis detects the conjugate wave $1-\left|\mathrm{s}_{3}(\mathrm{z}=\mathrm{L}, \mathrm{t})\right|$.

Figure 6a1 displays the input $\mathrm{s}_{3}$ set/reset control spikes imprinted on the 10-Gbit/s signal and detected on the low bandwidth oscilloscope beyond the PBS. No polarization segregation occurs and thus the eye-diagram (a2) presents a combination of both polarization PBS channels. At the opposite, when the average power is increased to $570 \mathrm{~mW}(27.5$ $\mathrm{dBm}$ ), the combined effects of polarization digitalization and associated hysteresis cycle make switch the whole energy of the optical data from one axis of the PBS to the other with an excellent discrimination. Remarkably, the output polarization does not depend on its current input value but also on its past value. Indeed, the switch remains in its last state until an erasing polarization trigger is sent to the system, thus demonstrating the all-optical remote processing capability of that system. As a result, we can observe on both axis of the PBS in Figs. 6b1 and $6 \mathrm{c} 1$ the appearance of conjugate data packets corresponding to the set/reset initial sequence. Finally, the 10-Gbit/s eyediagrams displayed in Fig. $6 \mathrm{~b} 2$ and $6 \mathrm{c} 2$ show a wide opening as well as a high polarization digitalization efficiency with an extinction ratio between each orthogonal axis above $20 \mathrm{~dB}$.

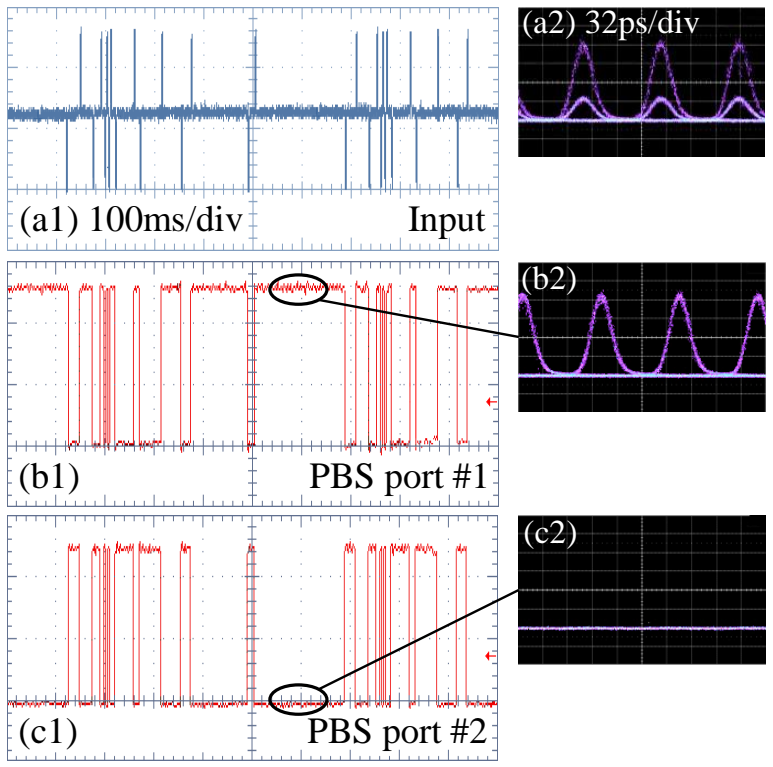

Fig. 6. Experimental proof-of-principle of data-packet switching operation based on the hysteresis properties of the Omnipolarizer. Parameters of the system are the same as in Fig. 4. The 10-Gbit/s RZ input signal has an average power of $27.5 \mathrm{dBm}$. The left column displays the temporal evolution of the intensity profile recorded beyond a PBS and monitored on a low-bandwidth oscilloscope (a) Input set/reset $\mathrm{s}_{3}(\mathrm{z}=0, \mathrm{t})$ spike sequence (b, c) Output intensity profile on port\#1 and port\#2 of the PBS (a2, b2 and c2) Corresponding 10Gbit/s eye-diagrams.

Finally, as noticed in Sec .III, for the typical fibers undertest which are few-km long, let us say $\mathrm{L}=4 \mathrm{~km}$, and whose Kerr coefficient is $\gamma \approx 2 \mathrm{~W}^{-1} \mathrm{~km}^{-1}$, signal power values $>500 \mathrm{~mW}$ allow reaching a strong nonlinear regime which corresponds to 
truly effective flip-flop and switching operations. Larger input powers can improve the device performance, but on the other hand this performance grows asymptotically with power: consequently, using powers much larger than $500 \mathrm{~mW}$ do not bring real and evident benefits with respect to the use of more moderate powers.

\section{All-Optical Polarization AlignMENT}

The capability of all-optically aligning the polarization of an incoming signal towards a unique fixed SOP, in such a way to implement an ideal polarizer, is of fundamental and practical interests which could find numerous applications in photonics. For instance, an all-optical repolarization functionality could be a powerful processing for the implementation of polarization sensitive devices such as silicon based high-contrast integrated optics or photonic-crystal waveguides for future transparent networks. For that ultimate purpose, we then discuss in this section how the Omnipolarizer may work as a polarization funnel and we illustrate the experimental proof-of-principle of a $10 \mathrm{Gbit} / \mathrm{s}$ polarization alignment for telecom applications. To this aim, the Omnipolarizer is implemented in its active configuration, involving the amplified fiber loop setup described in Fig. 3.

As in the case of the bistable regime discussed in the previous section, the starting point is represented by the stable stationary solutions of Eqs. (1). On the other hand, in this case the active setup makes the reflection coefficient $\rho$ to be larger than unity and $\mathrm{R}$ to be in general a not-identity matrix. This makes the analysis of the system to be considerably more complex than in the case $\rho=1$ and $R=\operatorname{diag}(1,1,1)$.

However extensive numerical computations of the spatiotemporal solutions of Eqs.(1) provide a global overview on the full dynamics of the system. The main notion that should be retained is that, differently from the bistable regime, once that $\mathrm{R}, \mathrm{N}$ and $\rho$ are fixed the output value $\mathbf{s}(\mathrm{z}=\mathrm{L})$ of the stable stationary states is nearly the same whatever the input value $\mathbf{s}(\mathrm{z}=0)$ is. Indeed a polarization rotation in the reflective loop, which corresponds to $\mathrm{R} \neq \operatorname{diag}(1,1,1)$, breaks the symmetry of the two pole attractors and leads to a concentration of the output SOP in a unique area of attraction. The choice of the surviving output SOP is here mainly determined by the symmetry breaking introduced by the rotation matrix R. Consequently, in this regime, any input SOP injected in the fiber is attracted in output towards a unique and fixed SOP which depends on R, N and $\rho$. Furthermore, systematic numerical simulations show that the more $\rho$ increases in the range $1.2 \leq \rho \leq 2$, the stronger this attraction is.

In order to test the performances of our device in a telecom environment, we experimentally detected the distribution of the output SOP over the Poincaré sphere for different values of $\rho$ and for an input 10-Gbit/s RZ signal. The input signal is the same as in the experiments reported above and is injected into the Omnipolarizer with a fixed average power of $27 \mathrm{dBm}(500$ $\mathrm{mW})$. The fiber implemented into the device is a $6.2 \mathrm{~km}$-long NZDSF fiber characterized by a chromatic dispersion of -1.5 $\mathrm{ps} / \mathrm{nm} / \mathrm{km}$ at $1550 \mathrm{~nm}$, a Kerr coefficient of $\gamma=1.7 \mathrm{~W}^{-1} \mathrm{~km}^{-1}$, a PMD coefficient of $0.05 \mathrm{ps} / \mathrm{km}^{1 / 2}$ and linear losses of 0.2
$\mathrm{dB} / \mathrm{km}$. Before injection into the device, the polarization state of the 10-Gbit/s input signal is first genuinely scrambled by means of an opto-electronic polarization scrambler (PS) at a rate of $0.5 \mathrm{kHz}$ so that its $\mathrm{SOP}$ distribution covers the entire Poincaré sphere (Fig. 7a). Figure 7 depicts the evolution of the state-of-polarization of the 10-Gbit/s signal recorded at the output of the Omnipolarizer as a function of the reflective coefficient $\rho$, i.e. the backward power. When $\rho \leq 1$ (Fig. 7b-c), similarly to the previous bistability experiments, we notice a polarization attraction towards both the poles of the sphere. However, in the active configuration, when $\rho>1$ an outstanding attraction towards the north pole of the sphere takes place: when $\rho=1.5$ (Fig. 7f), corresponding to a backward power of 27.5 $\mathrm{dBm}$. In this case, all the output SOPs are close to the north pole, which proves the efficient polarization alignment of the initially depolarized signal.
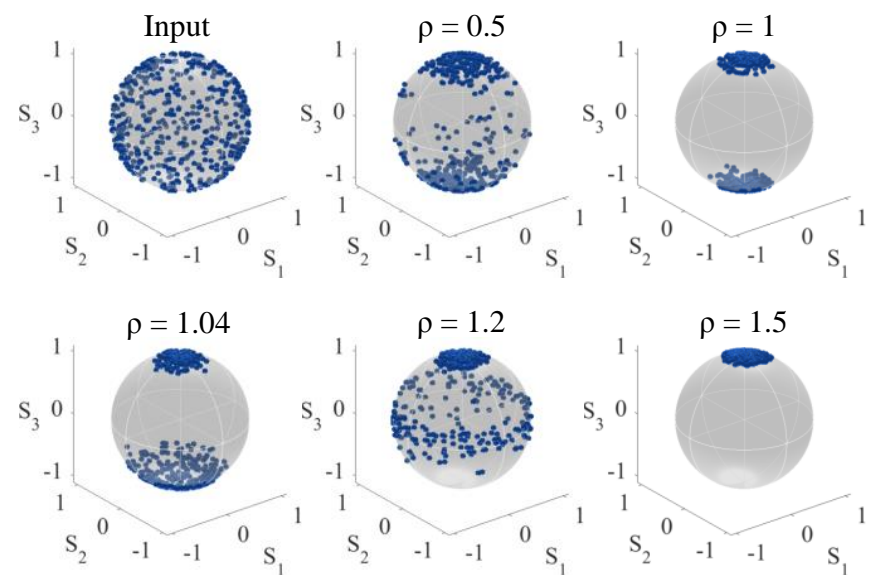

Fig. 7. Experimental evolution of the 10-Gbit/s signal SOP distribution recorded at the output of the Omnipolarizer for different values of the reflection coefficient $\rho$. The input signal is initially fully scrambled over the Poincaré sphere, panel (a), and has a fixed input average power of $27 \mathrm{dBm}$.

Note that in this example the pool of attraction is centered on the north pole because the polarization rotation, described by the matrix R and imposed by the amplified reflective loop setup is equivalent to a positive rotation around the direction of $\mathrm{s}_{3}$. A negative rotation around the same direction would generate an attraction towards the south pole, instead. But, more generally, if the rotation is around a different direction then the attraction SOP will be a particular point over the sphere which is neither the north pole nor the south pole (see for example Fig. 1b). As a result, we can simply tune the tracking SOP by controlling the rotation by means of the polarization controller inserted within the loop.

Besides the visual representation of the output SOP distribution over the Poincaré sphere, an important quantitative parameter which measures the strength of the alignment process is represented by the degree of polarization (DOP), defined as:

$$
\operatorname{DOP}(\mathrm{z})=\left(\left\langle\mathrm{s}_{1}(\mathrm{z}, \mathrm{t})\right\rangle^{2}+\left\langle\mathrm{s}_{2}(\mathrm{z}, \mathrm{t})\right\rangle^{2}+\left\langle\mathrm{s}_{3}(\mathrm{z}, \mathrm{t})\right\rangle^{2}\right)^{1 / 2}
$$

where the brackets \langle\rangle denote a temporal averaging. A depolarized signal which is uniformly scrambled all over the Poincaré sphere, as the input in Fig. 7a, has a DOP close to 0 . Note however that according to the definition given in Eq.(2), also the bistable output displayed in Fig. 7c has a DOP close to 
0 , because its SOP samples are localized around 2 opposite attraction poles. On the other hand, when the attraction SOP is unique, as it is the case of Fig. 7f, then the DOP is nearly unitary. Therefore, the DOP of the output signal as function of $\rho$ typically exhibits the evolution illustrated in Fig. 8a: it is close to 0 when $0.8 \leq \rho \leq 1$, namely in the bistable regime, but after that it rapidly grows towards 1 and it remains nearly unitary for $1.2 \leq \rho \leq 2$, namely in the alignment regime. For values of $\rho$ beyond 2, not displayed in Fig. 8a, the system gradually loses its attraction capability as it begins to exhibit a complex chaotic dynamics, which compromises the polarization alignment towards a unique SOP. This issue will be discussed in the next section.

In order to quantify the performances of the polarization alignment process upon a Telecom signal in the time domain, we monitored the eye-diagram of the 10-Gbits/ RZ signal undertest behind a polarizer at the input and output of the Omnipolarizer. The results are displayed in Fig. 8b. The input signal, which is completely depolarized (see the sphere in Fig. 7a), exhibits a completely closed eye-diagram (Fig. 8b, upper panel). Indeed, due to the initial scrambling process, all the polarization fluctuations are thus transformed into intensity fluctuations through the polarizer. On the other hand, at the output of the Omnipolarizer, when the backward power is set to $27.5 \mathrm{dBm}$, corresponding to the case $\rho=1.5$ of Fig. $7 \mathrm{f}$, the attraction process acts in full strength leading to an efficient polarization alignment and thus a wide opened output eyediagram (Fig. 8b, lower panel).

These results remarkably confirm the high efficiency of the repolarization process performed by the Omnipolarizer. We stress that this outcome represents a truly key breakthrough advance for practical usage of nonlinear polarizers in telecom applications. Indeed, despite the first proof-of-principle of polarization alignment was proposed more than a decade ago exploiting the nonlinear interaction between a signal and a counter-propagative independent pump in an isotropic fiber [41], in the present case, the independent pump beam is not required: the signal self-organizes its own SOP and the fiber is a standard $\mathrm{km}$-long telecom fiber. This proves the unexpected robustness of the nonlinear polarization alignment even in presence of local anisotropy and randomly varying birefringence. Note that similar results have been obtained at 40 $\mathrm{Gbit} / \mathrm{s}$ and show that very weak penalties in the bit-error-rate measurements are induced by the repolarization process [56].
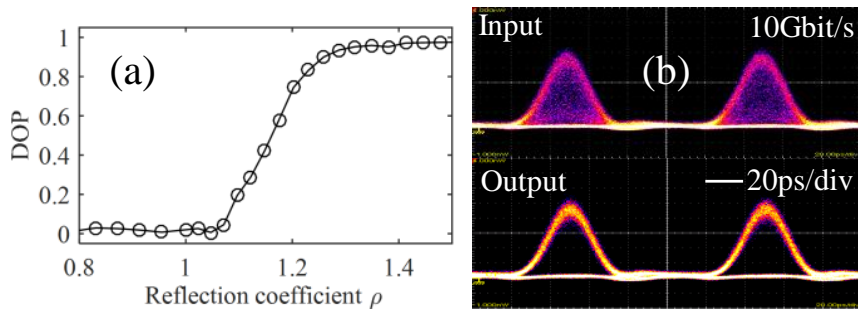

Fig. 8. (a) Experimental output DOP versus $\rho$ for a $10 \mathrm{Gbit} / \mathrm{s}$ RZ signal transmitted into the Omnipolarizer, the input average power is $27 \mathrm{dBm}$ (b) Eyediagram of the 10-Gbit/s RZ signal recorded beyond a polarizer at the input of the system (upper panel) and at the output of the Omnipolarizer when $\rho=1.5$ (lower panel).
We highlight that, similarly to the case of the bistability regime, for the typical employed fibers which are few-km long, let us say $\mathrm{L}=6 \mathrm{~km}$, and whose Kerr coefficient is $\gamma \approx 2 \mathrm{~W}^{-1} \mathrm{~km}^{-1}$, signal power values $>500 \mathrm{~mW}$ allow reaching a strong nonlinear regime where SOP alignment is very efficient.

\section{All-Optical ChaOtic POLARIZATION SCRAMBleR}

In this section we discuss the third regime of operation of the Omnipolarizer, which allows implementing an all-optical fully chaotic polarization scrambler. Indeed, as underlined in Sections II and III, for a large power imbalance between the forward beam and its backward replica, the system exhibits a genuine chaotic dynamics so that even a well-polarized incident signal becomes scrambled all over the Poincare sphere at the fiber output [64-69].

Two threshold values of the reflection coefficient $\rho$ are found that characterize this chaotic dynamics, which are $\rho_{\mathrm{A}}=9 \pi /$ $\left(16 \cdot L \cdot P_{s, \text { out }} \gamma\right)$ and $\rho_{C} \simeq 5 \rho_{A}$, being $P_{s, \text { out }}$ the power of the forward signal at the fiber exit. If $\rho$ is below the threshold $\rho_{\mathrm{A}}$ then the system exhibits stationary states that are stable, therefore a signal which is fully polarized in input $(\mathrm{DOP}=1)$ turns out to be still polarized in output with an SOP that strictly depend on the system parameters and on the input SOP [69]. On the other hand when $\rho>\rho_{C}$ the stationary states don't exhibit anymore a monotonic evolution along the fiber length and thus, start to oscillate and become unstable. As a result, the signal cannot be attracted towards any stable state: the output SOP varies endlessly in time and becomes temporally scrambled and therefore depolarized at the fiber output (see Fig. 1c). More precisely, the output polarization turns out to be not only randomly varying in time but also chaotic, which is attested by a positive Lyapunov coefficient, calculated as in ref. [72]. For intermediate values $\rho_{A} \leq \rho \leq \rho_{C}$ the output signal undergoes a complex dynamics: depending on its input polarization and on the rotation R, it may be fully polarized or scrambled or even exhibit a periodic temporal trajectory [69].

Therefore, a genuine scrambling regime is reached whenever $\rho>\rho_{C}$. In this regime our device behaves as an efficient polarization scrambler. The condition $\rho>\rho_{C}$ can be read as $P_{s, \text { out }}{ }^{\circ}$ $\rho>45 \pi /(16 L \gamma)$ : considering as usual a reference fiber with $\gamma$ $=2 \mathrm{~W}^{-1} \mathrm{Km}^{-1}$ and $\mathrm{L}=4 \mathrm{~km}$, we find $\rho \mathrm{P}_{\mathrm{s}, \text { out }}>1.1 \mathrm{~W}$, where $\rho \mathrm{P}_{\mathrm{s} \text {,out }}$ is the total backward power $\mathrm{P}_{\mathrm{J}, \text { out. }} \mathrm{A}$ backward power in the order of $1 \mathrm{~W}$ is thus required to reach the chaotic regime in the standard fibers employed in experiments.

An important parameter for the evaluation of the scrambling efficiency is represented by the scrambling speed $\mathrm{v}=\langle|\partial \mathrm{t} \mathrm{s}|\rangle$, which defines the average angle covered by the Stokes vector $\mathrm{s}$ in 1 second over the Poincaré sphere [57]. In order to estimate $\mathrm{v}$, we have collected a series of samples of $\mathrm{s}$ at the output of the fiber at a rate of nearly 1 microsecond. Finally, from the sequence of samples we have estimated the speed according to the aforementioned formula. Being that typical speeds are in the order of hundreds of $\mathrm{KHz}$, to whom it corresponds a coherence time of several microseconds, a sampling rate of 1 microsecond turned out to be appropriate for a correct measurement. Interestingly enough, the scrambling speed $\mathrm{v}$ is directly 
proportional to the reflection coefficient $\rho$, which is thus the tunable parameter in order to control the scrambling performances. Furthermore, the time scale of the output polarization fluctuations is well estimated by $1 / \mathrm{v}$.

In order to explore the scrambling regime, we have carried out a first series of experiments by considering the initial polarized $100-\mathrm{GHz}$ incoherent wave with a fixed arbitrary polarization state $(\mathrm{DOP}=1)$ injected into the Omnipolarizer with a constant power of $15 \mathrm{dBm}$. The fiber implemented into the device is a $5.3 \mathrm{~km}$-long NZ-DSF and is characterized by linear losses of $0.24 \mathrm{~dB} / \mathrm{km}$ and a nonlinear coefficient $\gamma=1.7$ $\mathrm{W}^{-1} \mathrm{~km}^{-1}$. According to these parameters, the thresholds are respectively $\rho_{A} \simeq 8$ and $\rho_{C}=\simeq 40$.

In Fig. 9a we show the experimental DOP as function of $\rho$. In good agreement with the estimations of $\rho_{A}$ and $\rho_{C}$, we observe that below a reflection coefficient close to $\rho=8$, corresponding to a backward power of $23 \mathrm{dBm}$, the output DOP remains nearly unitary because the output signal is still fully polarized. Conversely, for values of $\rho>40$, corresponding to a backward power larger than $30 \mathrm{dBm}$, the system enters into the genuine scrambling regime. The experimental DOP then drops to much lower values, typically below 0.3 , corresponding to an efficient scrambling of the output SOP covering the whole Poincaré sphere (see Fig. 9d). It is also noteworthy that in this regime the Lyapunov coefficient of the output SOP, evaluated upon the procedure described in ref. [72] and shown in Fig. 9b, is found to be strictly positive, which provides a key signature of the chaotic nature of this scrambling dynamics. Furthermore, as previously underlined and shown in Fig. 9c, the scrambling speed is almost directly proportional to $\rho$. Indeed, based on intensive numerical simulations, we have found that this speed is almost directly proportional to the input signal power as well to $\rho$.

We stress that the experimental results show that our device is mainly limited by propagation losses and by the detrimental Rayleigh back-scattering when large amplification factors are used. These deleterious effects practically limit the scrambling speed of our system around $400 \mathrm{krad} / \mathrm{s}$. Note however that this value matches the scale of fast polarization changes encountered in high-speed fiber optic systems due to surroundings variations (temperature fluctuations, mechanical stress) [6]: the output scrambled signal from the Omnipolarizer may thus be employed to emulate polarization fluctuations undergone by a signal which is transmitted in a real fiber system, which makes the proposed chaotic regime of practical interest.

Note also in Fig. 9 the excellent agreement between experimental results and numerical results obtained from solution of Eqs. (1) using the experimental parameters, which is a further confirmation of the validity of Eqs. (1).
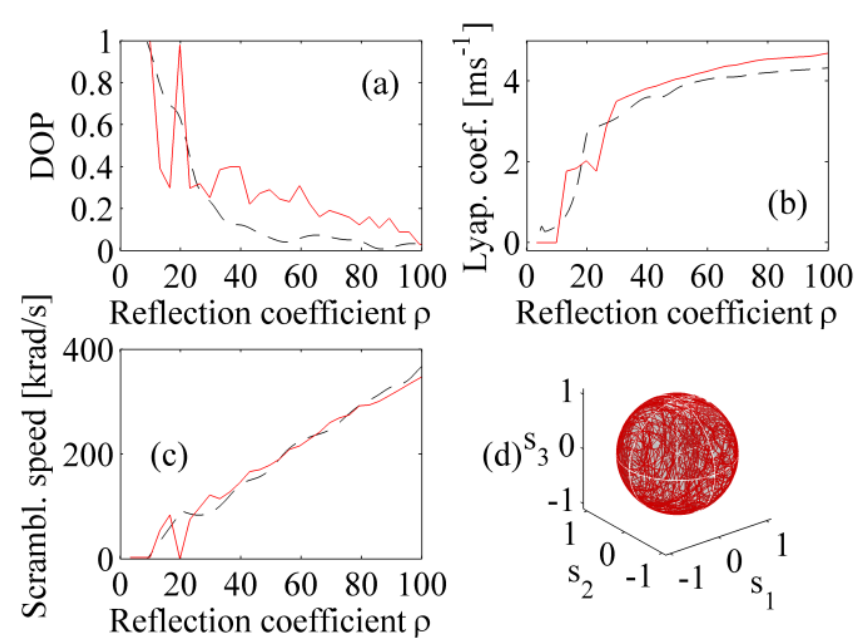

Fig. 9. Scrambling performances when using a NZ-DSF $5.3 \mathrm{~km}$-long fiber characterized by linear losses of $0.24 \mathrm{~dB} / \mathrm{km}$ and a nonlinear coefficient $\gamma=1.7$ $\mathrm{W}^{-1} \mathrm{~km}^{-1}$. The input power is set to $15 \mathrm{dBm}$. Experimental results (red solid lines) are compared to numerical results (black dotted lines). (a) DOP of the output signal as a function of the reflective coefficient $\rho$. (b) Corresponding Lyapunov coefficient. (c) Corresponding scrambling speed. (d) Distribution of the output SOP when $\rho=60$.

In order to evaluate the potential of this all-optical polarization scrambler for Telecom signal processing, we experimentally implemented our device into a wavelength division multiplexing transmission. To this aim, the initial incoherent signal was then replaced by the following WDM experimental source depicted in Fig. 10. A 10-GHz modelocked fiber laser delivering 2-ps pulses at $1551 \mathrm{~nm}$ is first encoded at $10 \mathrm{Gbit} / \mathrm{s}$ under an OOK RZ modulation format and next amplified up to $30 \mathrm{dBm}$. A supercontinuum is then generated within a 500-m long dispersion-flattened highly nonlinear fiber (DF-HNLF) characterized at the pump wavelength by a chromatic dispersion of $-1 \mathrm{ps} / \mathrm{nm} / \mathrm{km}$, a dispersion slope of $0.006 \mathrm{ps}^{2} / \mathrm{nm} / \mathrm{km}$, fiber losses of $0.6 \mathrm{~dB} / \mathrm{km}$ and a Kerr coefficient of $10.5 \mathrm{~W}^{-1} \cdot \mathrm{km}^{-1}$. Five $10-\mathrm{Gbit} / \mathrm{s}, 12-\mathrm{GHz}$ bandwidth, WDM channels centered at 1540.2 (C1), 1542 (C2), 1543.45 (C3), 1545 (C4) and $1546.2 \mathrm{~nm}$ (C5), and an additional pump channel, centered at $1550 \mathrm{~nm}$, are finally sliced into this resulting continuum thanks to a programmable optical filter (Waveshaper WS). All the channels are then decorrelated both in the time and polarization domains by means of a couple of demultiplexer/multiplexer with different paths and polarization rotations for each channel.

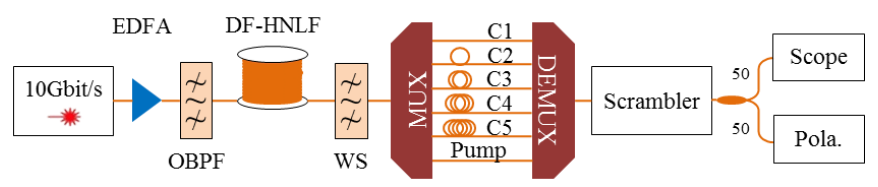

Fig. 10. Experimental setup of the 10-Gbit/s WDM source for testing the chaotic polarization scrambler in WDM configuration.

The resulting 10-Gbit/s WDM grid is injected into the Omnipolarizer with a fixed average power of $15 \mathrm{dBm}$. The key ingredient here is that a $100-\mathrm{GHz}$ optical bandpass filter is inserted into the reflective-loop in order to only counter- 
propagate the 1550-nm pump channel as a backward signal. Meanwhile, the five WDM channels are picked out of the device for analysis. The purpose of this spectral filtering is twofold: on the one hand, a single signal counter-propagates and thus imposes a unique and efficient scrambling process for all the transmitted channels. On the other hand, because of the strong level of power used for the backward signal, this frequency offset pump channel limits the deleterious impact of back Rayleigh scattering on the 5 other transmitted channels. At the output of the Omnipolarizer, each of the five WDM channels is characterized in polarization and in the time domain by means of an eye-diagram monitoring and bit-error-rate measurements.

Figures 11a-c display the output Poincaré spheres for 3 different WDM channels, corresponding to $\mathrm{C} 1, \mathrm{C} 3$ and $\mathrm{C} 5$, respectively. Note that the 2 other channels localized at $\mathrm{C} 2$ and $\mathrm{C} 4$ exhibit similar behaviors. For these measurements, the total input power is kept constant to $15 \mathrm{dBm}$ and only the $1550-\mathrm{nm}$ pump channel is back-reflected with an average power of 29 $\mathrm{dBm}$.

Quite remarkably, in spite of the fact that all the channels are initially decorrelated and enter into the system with different fixed SOPs, the device is still able to randomize the polarization of the whole WDM grid. Indeed, it is noteworthy in Fig. 11 that the SOP of each channel well covers the entire Poincaré sphere and is characterized by a low value of DOP below 0.2. Moreover, we have found that the polarization trajectories undergone by all the individual channels are correlated in time and characterized by roughly the same scrambling speed close to $140 \mathrm{krad} / \mathrm{s}$, thus confirming that the chaotic dynamics is here clearly imposed by the counter-propagating pump wave onto the other channels [69]. It is also important to notice that the scrambling speed reached by all the WDM channels are close to the one measured in the previous single channel configuration for similar involved average powers. Indeed, this all-optical scrambler is mainly sensitive to the average power of the counter-propagative beam.
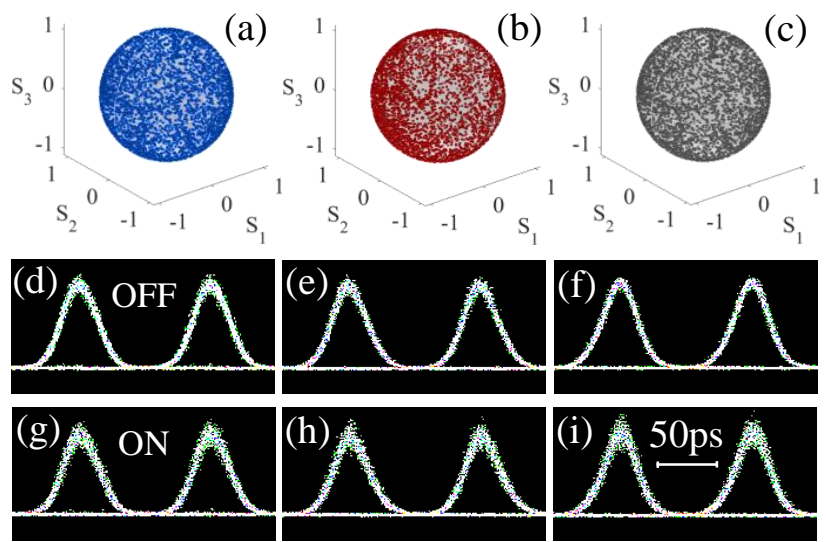

Fig. 11. WDM performances of the Omnipolarizer in the scrambling regime (ac) Output Poincaré spheres of the all-optical scrambler for channels C1, C3 and $\mathrm{C} 5$, respectively. The input power is fixed to $15 \mathrm{dBm}$ and the reflected 1550 $\mathrm{nm}$ pump channel is amplified to $29 \mathrm{dBm}(\mathrm{d}-\mathrm{i})$ Corresponding eye-diagrams in absence of backward signal (pump off) and when the backward 1550-nm pump is $\mathrm{ON}(\mathrm{j})$
Note that in these experiments, and in accordance with what previously exposed in Section III, despite the chaotic dynamics imposed on the output SOP the output temporal intensity profile is by the way greatly preserved, enabling high bit rate signal processing. This feature is well illustrated in Fig. 11(d-i) by monitoring the output 10-Gbit/s eye-diagrams. In particular, the upper row of insets (d-f) shows the initial high quality of the output eye-diagrams when the backward pump channel is switched off. At the opposite, the bottom row reports the corresponding eye-diagrams when the backward pump channel is now switched on at an average power of $29 \mathrm{dBm}$, so that the scrambling process can then operate efficiently. One can observe that the eye-diagram is outstandingly preserved with a wide opening and minor intensity degradations. A slight degradation of the temporal profiles, especially an increase of the amount of amplitude jitter can yet be observed. We attribute these impairments to the Rayleigh back-scattering provided by the spectrally broadened backward pump channel as well as to a weak Raman depletion effect caused by the pump onto the signal.

Finally, the impairments induced by the scrambling process on the whole 10-Gbit/s WDM grid have been quantified thanks to bit-error-rate measurements in passive mode (pump off) and pump on configurations. These measurements are summarized in Fig. 12 and show that low penalties are introduced by the scrambling process. Indeed, roughly $0.2 \mathrm{~dB}$ of power penalty have been estimated for the whole WDM channels at a BER of $10^{-9}$ when comparing the pump on/off configuration. Note that the power penalty of channel 1 in the back-to-back configuration is attributed to the degradation of its OSNR due to its carving into the initial continuum and to excess of spontaneous noise emission of our amplifier at this wavelength.

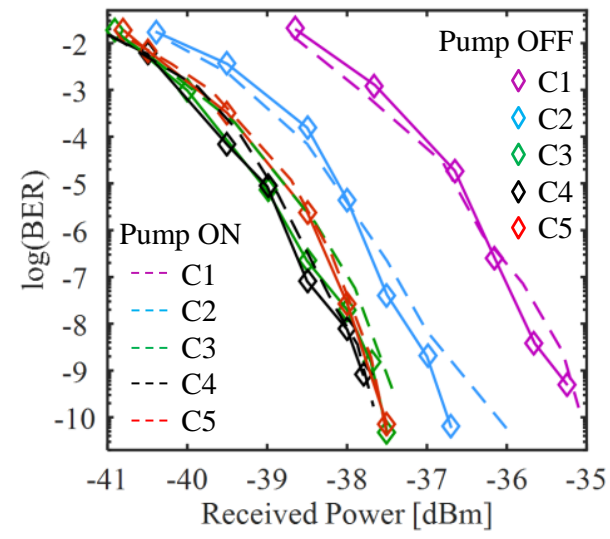

Fig. 12. Bit-error-rate measurements for the five 10-Gbit/s WDM channels propagating in the all-optical scrambler when the backward pump channel if turned off (solid lines) and in scrambling regime (pump on, dashed-line).

\section{CONCLUSIONS}

In conclusion, we have reported a theoretical, numerical and experimental description of a self-organization phenomenon of light state-of-polarization within a device called Omnipolarizer. The principle of operation of that system is based on a nonlinear cross-polarization interaction occurring in a km-long telecom optical fiber between an incident signal and its own counter- 
propagating replica generated at the fiber output by means of a reflective element. Depending on the power ratio between the forward and backward waves, three distinct operating regimes have been identified.

First of all, for a balance of powers just below unity (typically a reflective coefficient $\rho \sim 0.9$, forward and backward power of $27 \mathrm{dBm}$ and $25 \mathrm{dBm}$, respectively), the Omnipolarizer is characterized by a bistable behavior associated with a hysteresis cycle of the output state-of-polarization (bistable regime). Our theoretical predictions of the hysteresis are well confirmed by the experimental observations and show a high robustness, wide opening and sharp transitions in the cycle. This bistable regime has then been exploited in order to provide two proof-of-principles for optical signal processing. A polarization-based flip-flop optical memory, triggered by means of a remote sequence of set/reset polarization spikes imprinted onto an input continuous signal, has been successfully demonstrated. These results show that binary information can be perpetually stored or erased thanks to this all-optical digitalization of the output polarization. Therefore, the hysteresis property has also been exploited to demonstrate a 10-Gbit/s switching operation for data packets. Experimental monitoring of the eye-diagrams confirms the switching capability of our device along two orthogonal polarization channels with an extinction ratio larger than $20 \mathrm{~dB}$.

When the reflective coefficient is increased further above 1 , (typically $\rho \sim 1.5$, forward and backward power of $27 \mathrm{dBm}$ and $28 \mathrm{dBm}$, respectively) the present device turns out to act as a polarization funnel (alignment regime). Any input arbitrary polarization state is therefore aligned to a single output state without polarization depending loss. We have fully exploited this unique property in order to self-repolarize a 10-Gbit/s return-to-zero OOK signal with very weak degradation of the eye-diagram quality.

The third operating regime is basically based on a strong power unbalance between the two counter-propagating waves (scrambling regime: typically $\rho \sim 40$, forward and backward power of $15 \mathrm{dBm}$ and $30 \mathrm{dBm}$, respectively). Indeed, when the reflective coefficient of the Omnipolarizer exceeds a certain threshold the system exhibits a genuine chaotic dynamics for which the evolution of the output state-of-polarization of an initially well-polarized input signal becomes fully random. This nonlinear polarization scrambler has been successfully implemented on a 10-Gbit/s OOK WDM signal. In particular, we have experimentally shown that this device is able to simultaneously scramble the polarization of $5 \mathrm{WDM}$ channels with a scrambling speed around $140 \mathrm{krad} / \mathrm{s}$, whilst providing very low impairments on the signal quality. Moreover, the scrambling speed was found to be proportional to the reflective coefficient, which permits to tune the speed up to nearly 400 $\mathrm{krad} / \mathrm{s}$.

From a practical point of view, it is important to note that the nonlinear cross-polarization process involved in the Omnipolarizer is independent of wavelength and spectral bandwidth as already experimentally demonstrated in ref. [43] for the counter-propagating external pump wave polarization attraction configuration. The only requirement is the implementation of high power amplifiers centered at the signal wavelength. We could thus imagine to extend this device to the Ytterbium or Thulium bands around $1 \mu \mathrm{m}$ or $2 \mu \mathrm{m}$, respectively. Nevertheless, the counter-propagative configuration combined with the cross-polarization interaction of the Omnipolarizer imposes a strong nonlinear regime of propagation involving high levels of power, typically around $500 \mathrm{~mW}$, which is high above the standard telecommunication levels. Consequently, the Omnipolarizer could not be applicable to weak input signals and peculiar attentions have to be taken in order to avoid signal degradations. For instance, nonlinear pulse reshaping, such as Mamyshev regenerator, is required for high bit-rates return-to-zero signals so as to preserve the signal quality [74-78], while high level of nonlinear phase shift could be seen as a limitation for phase shift keying modulation formats and coherent detection. Moreover, it is important to note that dual polarization multiplexing formats are not compatible with the Omnipolarizer. Indeed, the self-polarization control is inherently based on a strong cross-talk among orthogonal polarizations, therefore two independent and orthogonal input channels would be unavoidably impaired at the fiber output. We could also note that a strong unpolarized input noise could degrade the polarization control performed by the Omnipolarizer; on the other hand the counter-propagative geometry guarantees that, for typical values of $15 \mathrm{~dB}<$ OSNR $<30 \mathrm{~dB}$, the Omnipolarizer efficiency is not impaired by the input noise [52].

Furthermore, despite the quasi-instantaneity of the Kerr nonlinear effect underlying within the Omnipolarizer, the response time of our device has been found to be much slower than the response time of the Kerr effect in silica. This is directly related to the counter-propagating nature and thus to the distributed interaction along the whole fiber length [46]. Hence a finite response time is necessary in order to establish a polarization equilibrium within the fiber under use. Practically, the time scale of the input polarization fluctuations which allows for an efficient bistable attraction or polarization alignment has been experimentally evaluated to a few microseconds. A similar time scale characterizes the output polarization fluctuations produced in the scrambling regime. In fact, the response time of the Omnipolarizer can be estimated of the order of the nonlinear time defined as $\mathrm{L}_{\mathrm{NL}} / \mathrm{c}$, which typically corresponds to a few microseconds with the parameters involved in our experiments. This analysis suggests that the response time of the Omnipolarizer could be reduced to sub-microsecond by implementing fibers with much higher Kerr coefficients, such as Chalcogenide, Tellurite, Bismuth or lead Silicate fibers [73-80]. In the same way the power employed could be reduced of one order of magnitude.

\section{ACKNOWLEDGEMENT}

This research was funded by the European Research Council under Grant Agreement 306633, ERC PETAL. https://www.facebook.com/petal.inside. We also thank the financial support of the Conseil Régional de Bourgogne in the frame work of the Photcom project and the Labex ACTION (ANR-11-LABX-0001-01). We warmly thank Drs. D. Sugny, H. R. Jauslin, G. Millot, S. Wabnitz, P. Morin and especially S. Pitois for fruitful discussions. 


\section{REFERENCES}

1. T. Geisler, "Low PMD Transmission Fibres," in European Conference on Optical Communications ECOC 2006, paper Mo.3.3.1, 2006.

2. A. J. Barlow, J. J. Ramskov-Hansen, and D. N. Payne "Birefringence and polarization mode-dispersion in spun singlemode fibers," Appl. Opt., vol. 20, pp. 2962-2968, 1981.

3. M. J. Li and D. A. Nolan, "Fiber spin-profile designs for producing fibers with low polarization mode dispersion," Opt. Lett., vol. 23, pp. 16591661, 1998.

4. L. Palmieri, "Polarization Properties of Spun Single-Mode Fibers," IEEE J. Lightw. Technol., vol. 24, pp. 4075-4088, 2006.

5. M. Boroditsky, M. Brodsky, N. J. Frigo, P. Magill, and H. Rosenfeldt, "Polarization dynamics in installed fiber optic systems" IEEE LEOS Annual Meeting Conference Proceedings (LEOS), pp. 413-414, 2005.

6. P. M. Krummrich and K. Kotten, "Extremely fast (microsecond scale) polarization changes in high speed long hail WDM transmission systems," in Proc. of Optical Fiber Commun. Conference, Los Angeles, USA, 2004.

7. S. C. Rashleigh, "Origins and control of polarization effect in single-mode fibers," IEEE J. Lightw. Technol., vol. 1, pp. 312-331, 1983.

8. R. Ulrich and A. Simon, "Polarization optics of twisted single-mode fibers, " Appl. Opt., vol. 18, pp. 2241-2251, 1979.

9. A. Simon and R. Ulrich, "Evolution of polarization along a single-mode fiber," Appl. Phys. Lett., vol. 31, pp. 517-520, 1977.

10. A. Galtarossa, L. Palmieri, M. Schiano, and T. Tambosso, "Measurement of birefringence correlation length in long single-mode fibers," Opt. Lett., vol. 26, pp. 962-964, 2001.

11. C. D. Poole, and R. E. Wagner, "Phenomenological approach to polarization dispersion in long single-mode fibers," Electron. Lett., vol. 22, pp. 1029-1030, 1986.

12. A. Galtarossa and C. R. Menyuk, Eds., Polarization Mode Dispersion. New York: Springer-Verlag, 2005.

13. J. Garnier, J. Fatome, and G. Le Meur, "Statistical analysis of pulse propagation driven by polarization-mode dispersion," J. Opt. Soc. Am. B, vol. 19, pp. 1968-1977, 2002.

14. P. K. A. Wai and C. R. Menyuk, "Polarization mode dispersion, decorrelation, and diffusion in optical fibers with randomly varying birefringence," IEEE J. Lightw. Technol., vol. 14, pp. 148-157, 1996.

15. A. Galtarossa, L. Palmieri, and L. Schenato, "Simplified phenomenological model for randomly birefringent strongly spun fibers," Opt. Lett., vol. 31, pp. 2275-2277, 2006.

16. N. Gisin, and B. Huttner, "Combined effects of polarization mode dispersion and polarization dependent losses in optical fibers," Opt. Commun., vol. 142, pp. 119-125, 1997.

17. D. Sperti, P. Serena and A. Bononi, "Optical solutions to improve PDMQPSK resilience against cross-channel nonlinearities: A Comparison," IEEE Photon. Technol. Lett., vol. 23, pp.667-669, 2011.

18. L. F. Mollenauer, J. P. Gordon, and F. Heismann, "Polarization scattering by soliton-soliton collisions," Opt. Lett., vol. 20, pp. 2060-2062, 1995.

19. J. Renaudier, G. Charlet, M. Salsi, O. B. Pardo, H. Mardoyan, P. Tran, and S. Bigo, "Linear Fiber Impairments Mitigation of 40-Gbit/s Polarization-Multiplexed QPSK by Digital Processing in a Coherent Receiver," IEEE J. Lightwave Technol., vol. 26, pp. 36-42, 2008.

20. G. Charlet "Coherent detection associated with digital signal processing for fiber optics communications," C. R. Physique, vol. 9, pp. 1012-1030, 2008.

21. G. Charlet, J. Renaudier, M. Salsi, H. Mardoyan, P. Tran, and S. Bigo, "Efficient Mitigation of Fiber Impairments in an Ultra-Long Haul Transmission of 40Gbit/s Polarization-Multiplexed Data, by Digital Processing in a Coherent Receiver," in Optical Fiber Communication Conference and Exposition, paper PDP17, 2007.

22. J. Hansryd, P. A. Andrekson, M. Westlund, J. Li, and P. Hedekvist, "Fibre-basedoptical parametric amplifiers and their applications," IEEE J. Sel. Top. Quantum Electron., vol. 8, pp. 506-520, 2002.

23. K. Inoue, "Polarization independent wavelength conversion using fiber four-wave mixing with two orthogonal pump lights of different frequencies,” IEEE J. Lightwave Technol., vol. 12, pp. 1916-1920, 1994.

24. K. K. Y. Wong, M. E. Marhic, K. Uesaka and Leonid G., Kazovsky, "Polarization-independent one-pump fiber-optical parametric amplifier," IEEE Photon Technol Lett., vol. 14, pp. 1506-1508, 2002.

25. H. Fukuda, K. Yamada, T.Tsuchizawa, T. Watanabe, H. Shinojima, and S. Itabashi, "Silicon photonic circuit with polarization diversity," Opt. Express, vol. 16, pp. 4872-4880, 2008.
26. M. Martinelli, P. Martelli, and S. M. Pietralunga, "Polarization stabilization in optical communications systems," IEEE J. Lightw. Technol., vol. 24, pp. 4172-4183, 2006.

27. B. Koch, R. Noe, D. Sandel, and V. Vitali Mirvoda, "Versatile endless optical polarization controller/tracker/demultiplexer," Opt. Express vol. 22, pp. 8259-8276, 2014.

28. B. Koch, R. Noe, V. Mirvoda, H. Griesser, S. Bayer, and H. Wernz "Record 59-krad/s Polarization Tracking in 112-Gb/s 640-km PDM-RZDQPSK Transmission," IEEE Photonics Technol. Lett., vol. 22, pp. $1407-1409,2010$.

29. W. H. J. Aarts and G. Khoe, "New endless polarization control method using three fiber squeezers," IEEE J. Lightwave Technol., vol. 7, pp. 1033-1043, 1989.

30. B. Koch, R. Noé, V. Mirvoda and D. and Sandel, "100-krad/s endless polarisation tracking with miniaturised module card," Electron. Lett., vol. 47, pp. 813-814, 2011.

31. E. Heebner, R. S. Bennink, R. W. Boyd, and R. A. Fisher "Conversion of unpolarized light to polarized light with greater than $50 \%$ efficiency by photorefractive two-beam coupling," Opt. Lett., vol. 25, pp. 257-259, 2000.

32. M. Martinelli, M. Cirigliano, M. Ferrario, L. Marazzi, and P. Martelli, "Evidence of Raman-induced polarization pulling," Opt. Express, vol. 17, pp. 947-955, 2009.

33. L. Ursini, M. Santagiustina, and L. Palmieri, "Raman Nonlinear Polarization Pulling in the Pump Depleted Regime in Randomly Birefringent Fibers," IEEE Photon. Technol. Lett., vol. 23, pp. 1041-1135, 2011.

34. A. Galtarossa, L. Palmieri, M. Santagiustina, L. Schenato, and L. Ursini, "Polarized Brillouin Amplification in Randomly Birefringent and Unidirectionally Spun Fibers," IEEE Photon. Technol. Lett., vol. 20, pp. 1420-1422, 2008.

35. N. J. Muga, M. F. S. Ferreira, and A. N. Pinto "Broadband polarization pulling using Raman amplification,” Opt. Express, vol. 19, pp. 1870718712, 2011.

36. V. Kozlov, J. Nuno, J. D. Ania-Castanon and S. Wabnitz, "Theoretical study of optical fiber Raman polarizers with counterpropagating beams," IEEE J. Lightwave Technol., Vol. 29, pp. 341-347, 2011.

37. L. Thevenaz, A. Zadok, A. Eyal and M. Tur, "All-optical polarization control through Brillouin amplification", in Optical Fiber Communication Conference, OFC'08, paper OML7 (2008).

38. Z. Shmilovitch, N. Primerov, A. Zadok, A. Eyal, S. Chin, L. Thevenaz, and M. Tur "Dual-pump push-pull polarization control using stimulated Brillouin scattering," Opt. Express, vol. 19, pp. 25873-25880, 2011.

39. B. Stiller, P. Morin, D. M. Nguyen, J. Fatome, S. Pitois, E. Lantz, H. Maillotte, C. R. Menyuk, and T. Sylvestre, "Demonstration of polarization pulling using a fiber-optic parametric amplifier," Opt. Express, vol. 20, pp. 27248-27253, 2012.

40. M. Guasoni, V. Kozlov, and S. Wabnitz, "Theory of polarization attraction in parametric amplifiers based on telecommunication fibers," $J$. Opt. Soc. Am. B, vol. 29, pp. 2710-2720, 2012.

41. S. Pitois and M. Haelterman, "Optical fiber polarization funnel," Nonlinear Guided Waves and Their Applications NLGW'01, paper MC79-1, 2001

42. J. Fatome, S. Pitois, P. Morin, and G. Millot, "Observation of light-bylight polarization control and stabilization in optical fibre for telecommunication applications," Opt. Express, vol. 18, pp. 15311$15317,2010$.

43. J. Fatome, P. Morin, S. Pitois and G. Millot, "Light-by-Light Polarization Control of $10-\mathrm{Gb} / \mathrm{s} \mathrm{RZ}$ and NRZ Telecommunication Signals," IEEE J. Sel. Top. Quantum Electron, vol. 18, pp.621-628, 2012.

44. V. V. Kozlov, J. Nuno and S. Wabnitz, "Theory of lossless polarization attraction in telecommunication fibers," J. Opt. Soc. Am. B, vol. 28, pp. 100-108, 2011.

45. K. Turitsyn and S. Wabnitz, "Stability analysis of polarization attraction in optical fibers," Opt. Commun., vol. 307, pp. 62-66, 2013.

46. V. V. Kozlov, J. Fatome, P. Morin, S. Pitois, G. Millot and S. Wabnitz, "Nonlinear repolarization dynamics in optical fibers: transient polarization attraction," J. Opt. Soc. Am. B, vol. 28, pp. 1782-1791, 2011.

47. E. Assémat, S. Lagrange, A. Picozzi, H. R. Jauslin and D. Sugny, "Complete nonlinear polarization control in an optical fiber system," Opt. Lett. vol. 35, pp. 2025-2027, 2010.

48. S. Lagrange, D. Sugny,A. Picozzi, and H. R. Jauslin, "Singular tori as attractors of four-wave-interaction systems," Phys. Rev. E, vol. 81, 016202, 2010. 
49. E. Assémat, A. Picozzi, H. R. Jauslin and D. Sugny, "Hamiltonian tools for the analysis of optical polarization control," J. Opt. Soc. Am. B, vol. 29, pp. 559-571, 2012.

50. E. Assémat, D. Dargent, A. Picozzi, H. R. Jauslin and D. Sugny, "Polarization control in spun and telecommunication optical fibers," Opt. Lett., vol. 36, pp. 4038-4040, 2011.

51. P. Morin, J. Fatome, C. Finot, S. Pitois, R. Claveau, and G. Millot, "Alloptical nonlinear processing of both polarization state and intensity profile for $40 \mathrm{Gbit} / \mathrm{s}$ regeneration applications," Opt. Express, vol. 19, pp. 17158-17166, 2011.

52. M. Barozzi and A. Vannucci "Lossless polarization attraction of telecom signals: application to all-optical OSNR enhancement," J. Opt. Soc. Am. $B$, vol. 31, pp. 2712-2720, 2014.

53. V. Costa Ribeiro, R. S. Luis, J. M. D. Mendinueta, B. J. Puttnam, A. Shahpari, N. J. C. Muga, M. Lima, S. Shinada, N. Wada and A. Teixeira "All-Optical Packet Alignment Using Polarization Attraction Effect," IEEE Photon. Technol. Lett., vol. 27, pp. 541-544, 2015.

54. P. Morin, S. Pitois, and J. Fatome, "Simultaneous polarization attraction and Raman amplification of a light beam in optical fibers," J. Opt. Soc. Am. B, vol. 29, pp. 2046-2052, 2012.

55. S. Pitois, A. Picozzi, G. Millot, H.R. Jauslin, M. Haelterman, "Polarization and modal attractors in conservative counterpropagating four-wave interaction," Europhys. Lett., vol. 70, pp. 88-94, 2005.

56. J. Fatome, S. Pitois, P. Morin, D. Sugny, E. Assémat, A. Picozzi, H. R. Jauslin, G. Millot, V. V. Kozlov and S. Wabnitz, "A universal optical allfiber omnipolarizer," Sci. Rep., vol. 2, 938, 2012.

57. P. Y. Bony, M. Guasoni, P. Morin, D. Sugny, A. Picozzi, H. Jauslin, S. Pitois and J. Fatome, "Temporal spying and concealing process in fibreoptic data transmission systems through polarization bypass," Nat. Commun., vol. 5, pp. 5:4678, 2014.

58. P.-Y. Bony, M. Guasoni, E. Assémat, S. Pitois, D. Sugny, A. Picozzi, H. R. Jauslin and J. Fatome, "Optical flip-flop memory and data packet switching operation based on polarization bistability in a telecomunnication optical fiber," J. Opt. Soc. Am. B, vol. 30, pp. 23182325, 2013.

59. E. Lichtman, R. G. Waarts, and A. A. Friesem, "Stimulated Brillouin scattering excited by a modulated pump wave in single-mode fibers," IEEE J. Lightwave Technol, vol. 7, pp.171-174, 1989.

60. N. Yoshizawa and T.; Imai, "Stimulated Brillouin scattering suppression by means of applying strain distribution to fiber with cabling," IEEE J. Lightwave Technol, vol. 11, pp.1518-1522, 1993.

61. J. M. Chavez Boggio, J. D. Marconi, and H. L. Fragnito, "Experimental and Numerical Investigation of the SBS-Threshold Increase in an Optical Fiber by Applying Strain Distributions," IEEE J. Lightwave Technol., vol. 23, 3808-3814, 2005.

62. Y. Imai and N. Shimada, "Dependence of stimulated Brillouin scattering on temperature distribution in polarization-maintaining fibers," IEEE Photon. Technol. Lett, vol. 5, pp.1335-1337, 1993.

63. L. Gruner-Nielsen, D. Jakobsen, S. Herstrom, B. Palsdottir, S. Dasgupta, D. Richardson, C. Lundstrom, S. Olsson and P. Andrekson, "Brillouin suppressed highly nonlinear fibers," in 38th European Conference and Exhibition on Optical Communications, 2012.

64. A. L. Gaeta, R. W. Boyd, J. R. Ackerhalt, and P. W. Milonni, "Instabilities and chaos in the polarizations of counterpropagating light fields," Phys. Rev. Lett., vol. 58, pp. 2432-2435, 1987.

65. D. J. Gauthier, M. S. Malcuit and a. R. Boyd, "Polarization Instabilities of Counterpropagating Laser Beams in Sodium Vapor," Phys. Rev. Lett., vol. 61, pp. 1828-1830, 1987.

66. D. J. Gauthier, M. S. Malcuit, A. L. Gaeta, and R. W. Boyd, "Polarization bistability of counterpropagating laser beams," Phys. Rev. Lett., vol. 64, pp. 1721-1724, 1990.

67. S. Trillo and S. Wabnitz, "Intermittent spatial chaos in the polarization of counterpropagating beams in a birefringent optical fiber," Phys. Rev. A, vol. 36, pp. 3881-3884, 1987.

68. M. V. Tratnik and J. E. Sipe, "Nonlinear polarization dynamics. II. Counterpropagating-beam equations: New simple solutions and the possibilities for chaos," Phys. Rev. A, vol. 35, pp. 2976-2988, 1987.

69. M. Guasoni, P. Y. Bony, M. Gilles, A. Picozzi, and J. Fatome, "Fast and Chaotic Fiber-Based Nonlinear Polarization Scrambler," arXiv:1504.03221, 2015.

70. M. Guasoni, P.-Y. Bony, S. Pitois, D. Sugny, A. Picozzi, H.-R. Jauslin and J. Fatome, "Fast Polarization Scrambler Based on Chaotic Dynamics in Optical Fibers," in European Conference on Optical Communications ECOC 2014, paper Tu.1.4.5, 2014
71. G. P. Agrawal, Nonlinear Fiber Optics, 4th ed., (Academic Press, New York, 2007).

72. M. T. Rosenstein, J. J. Collins, and C. J. De Luca, "A practical method for calculating largest Lyapunov exponents from small data sets," Physica D, vol. 65, pp. 117-134, 1993.

73. J. H. V. Price et al, "Mid-IR supercontinuum generation from nonsilica microstructured optical fibers," IEEE J. Sel. Top. Quantum Electron., vol. 13, pp. 738-750, 2007.

74. P. V. Mamyshev, "All-optical data regeneration based on self-phase modulation effect," in European Conference on Optical Communication, ECOC'98, pp. 475-476, 1998.

75. M. Matsumoto, "Fiber-Based All-Optical Signal Regeneration," IEEE J. Sel. Top. Quant., vol. 18, pp. 738-752, 2012.

76. L. Provost, C. Finot, K. Mukasa, P. Petropoulos, and D. J. Richardson, "Design scaling rules for 2R-Optical Self-Phase Modulation-based regenerators 2R regeneration," Opt. Express, vol. 15, pp. 5100-5113, 2007.

77. M. Matsumoto, "Performance Analysis and Comparison of Optical 3R Regenerators Utilizing Self-Phase Modulation in Fibers," IEEE J. Lightw Technol., vol. 22, pp. 1472-1482, 2004.

78. C. Finot, T. N. Nguyen, J. Fatome, T. Chartier, S. Pitois, L. Bramerie, M. Gay, and J.-C. Simon, "Numerical study of an optical regenerator exploiting self-phase modulation and spectral offset filtering at $40 \mathrm{Gbit} / \mathrm{s}$," Opt. Commun., vol. 281, pp. 252-2264, 2008.

79. C. Rosenberg Petersen, U. Moller, I. Kubat, B. Zhou, S. Dupont, J. Ramsay, T. Benson, S. Sujecki, N. Abdel-Moneim, Z. Tang, D. Furniss, A. Seddon and O. Bang, "Mid-infrared supercontinuum covering the 1.4$13.3 \mu \mathrm{m}$ molecular fingerprint region using ultra-high NA chalcogenide step-index fibre," Nat. Photon., DOI: 10.1038, 2014.

80. O. Mouawad, J. Picot-Clémente, F. Amrani, C. Strutynski, J. Fatome, B. Kibler, F. Désévédavy, G. Gadret, J.-C. Jules, D. Deng, Y. Ohishi and F. Smektala, "Multioctave midinfrared supercontinuum generation in suspended-core chalcogenide fibers," Opt. Lett., vol. 39, pp. 2684-2687, 2014. 


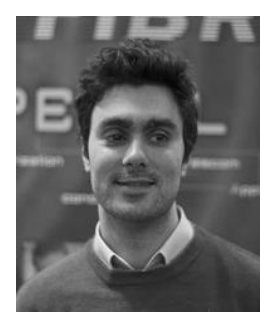

Massimiliano Guasoni was born in Verona, Italy, in 1982. He received the M.S. degree and the Ph.D. in Telecommunications from the University of Brescia (Italy) in 2007 and 2011, respectively, working on the subject of plasmonic waveguides. From 2010 to 2012 he was postdoctoral research fellow at the Department of Information Engineering of the University of Brescia (Italy), working on the subject of nonlinear crosspolarization interactions in photonic systems. Since 2013 he is postdoctoral research fellow at the Laboratoire Interdisciplinaire Carnot de Bourgogne of the University of Bourgogne, Dijon (France). His main research interests are nonlinear optics, plasmonics, computational electromagnetism and photonic structures.

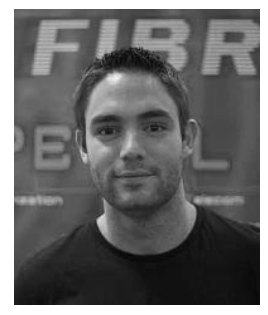

Pierre-Yves Bony was born in Langres, France, in 1989. After graduating with a scientific degree at Diderot high school of Langres and a DUT Mesures Physiques at the technological university institute (IUT) Léonard de Vinci of Reims, he got a Licence of Physics and then a Master Physique Laser \& Matériaux (PLM) at the University of Burgundy of Dijon. In 2012, he began a Doctorate of Physics at the Interdisciplinary Carnot of Burgundy Laboratory (ICB) of Dijon. His subject deals with the all-optical control of the light polarization in telecommunication optical fibers and the new applications based on this concept for new polarization shift keying format. His thesis is an integral part of the PETAL project directed by Julien Fatome.

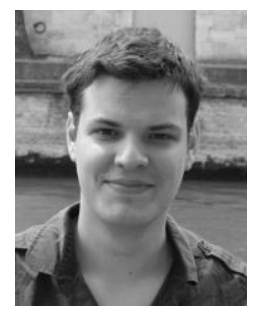

Marin Gilles was born in Hyères, France in 1991. He received a Bachelor degree in Electronics, Energy and Automatism in 2011 and a Master degree in nanotechnology in 2013. He currently is a Ph.D. student at University of Bourgogne, France in the Solitons, Lasers and Optical Communications team. His research interest is in the study of light polarization in optical fibers, how it can auto-organize within, the generation of solitonic structures and their propagation inside the fibers.

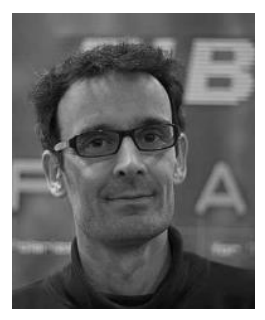

Antonio Picozzi was born in Monza, Italy, in 1971. He completed his studies at the University of Nice (France), including his $\mathrm{PhD}$ thesis in 1997 in the context of the resonant three-wave interaction modeling stimulated Brillouin or Raman effects. After a Post-Doc at ULB (Brussels) in which he studied the dynamics of quadratic nonlinear waves, he obtained in 2000 an associate Professor position at the University of Nice. At that time he started to work on nonlinear optics with partially coherent waves. Motivated by a close interaction with experimentalists, he obtained in 2005 a full research position at CNRS in the University of Burgundy (Dijon, France). In this way he was able to develop the theme of incoherent nonlinear optics through numerous collaborations as well as the supervision of $\mathrm{PhD}$ students and postdocs. This subject has reached a certain degree of maturation, especially with the development of a unified nonequilibrium thermodynamic formulation of statistical nonlinear optics (long-range wave turbulence in analogy with the Vlasov formulation of Gravitation, thermalization and condensation of nonlinear waves in analogy with the kinetic gas theory, spectral incoherent solitons in analogy with weak Langmuir turbulence in plasmas, or the breakdown of thermalization in analogy with the Fermi-PastaUlam problem). He co-authored more than 80 articles in peerreviewed journal, he has been invited to give more than 50 talks in international conferences and was promoted CNRS Research Director in 2013.

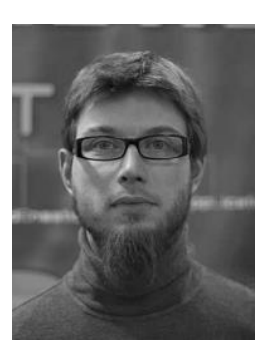

Julien Fatome was born in CharlevilleMézières, France, in 1978. After graduating from the engineer school ESIREM, he received the Master's degree in 2000 and the $\mathrm{Ph} . \mathrm{D}$. degree in physics for studies of ultrashort pulse propagation at $160-\mathrm{Gb} / \mathrm{s}$ in dispersion managed optical fiber lines in 2004, from the University of Bourgogne, Dijon, France. In 2005, he became a Research Engineer in the Centre National de la Recherche Scientifique (CNRS), Department of Physics, ICB, University of Bourgogne. He is currently carrying out research on nonlinear effects in optical fibers and ultrahigh bit rate optical communications, in particular on the all-optical control of light state of polarization. He has published over 160 contributions in peer-reviewed journals and conference proceedings and 4 patents. He is currently leading the ERC starting grant PETAL project. 